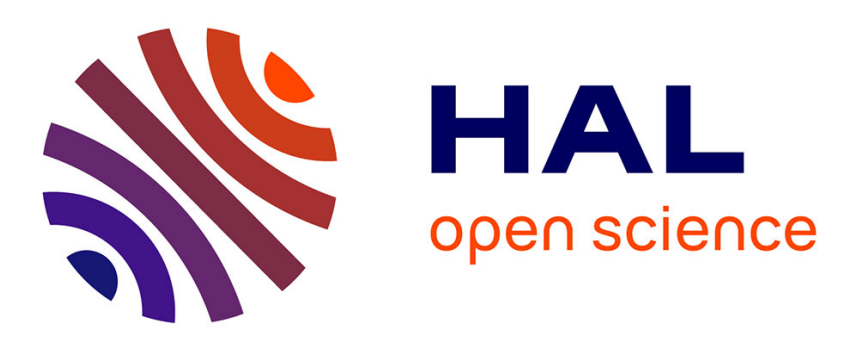

\title{
Familles de caractères de groupes de réflexions complexes
}

\author{
Gunter Malle, Raphaël Rouquier
}

\section{To cite this version:}

Gunter Malle, Raphaël Rouquier. Familles de caractères de groupes de réflexions complexes. 2003. hal-00000291v2

\section{HAL Id: hal-00000291 \\ https://hal.science/hal-00000291v2}

Preprint submitted on 10 Oct 2003

HAL is a multi-disciplinary open access archive for the deposit and dissemination of scientific research documents, whether they are published or not. The documents may come from teaching and research institutions in France or abroad, or from public or private research centers.
L'archive ouverte pluridisciplinaire HAL, est destinée au dépôt et à la diffusion de documents scientifiques de niveau recherche, publiés ou non, émanant des établissements d'enseignement et de recherche français ou étrangers, des laboratoires publics ou privés. 


\title{
FAMILLES DE CARACTÈRES DE GROUPES DE RÉFLEXIONS COMPLEXES
}

\author{
GUNTER MALLE AND RAPHAËL ROUQUIER
}

\begin{abstract}
RÉSUMÉ. Nous étudions certains types de blocs d'algèbres de Hecke associées aux groupes de réflexions complexes qui généralisent les familles de caractères définies par Lusztig pour les groupes de Weyl. Nous déterminons ces blocs pour les groupes de réflexions spetsiaux et nous établissons un théorème de compatibilité entre familles et $d$-séries de Harish-Chandra.
\end{abstract}

\section{INTRODUCTION}

Lusztig a construit une partition des caractères irréductibles d'un groupe de Coxeter fini $W$ en familles, à l'aide de la théorie des cellules. Cette partition apparaît naturellement dans le paramétrage de Lusztig des caractères unipotents d'un groupe réductif sur un corps fini.

Gyoja [13] a démontré (cas par cas) que la partition en " $p$-blocs" de l'algèbre de Iwahori-Hecke affine la partition en familles et que les deux partitions cö̈ncident lorsque $p$ est l'unique mauvais nombre premier. Dans [22], il a ensuite été démontré (par un argument général utilisant l'algèbre $J$ de Lusztig et une propriété des anneaux basés) que les blocs de l'algèbre d'Iwahori-Hecke sur un anneau convenable $\mathcal{O}$ coïncident avec les familles.

Les groupes de Weyl finis forment une classe particulière de groupes de réflexions complexes. Des travaux récents ont apportés de nombreux indices que les groupes de réflexions complexes (au moins certains d'entre eux) jouissent de propriétés analogues aux groupes de Weyl (voir par exemple [7, 19]). On dispose en particulier d'algèbres du type Iwahori-Hecke. Pour le moment, il n'existe pas de définition de cellules pour les groupes de réflexions complexes. On ne peut donc utiliser l'approche de Lusztig pour définir les familles de caractères, mais celle de [22] décrite plus haut est differente : on définit les familles comme blocs de l'algèbre de Iwahori-Hecke sur un certain anneau $\mathcal{O}$.

La première partie de cet article consiste en la détermination, cas par cas, des $\mathcal{O}_{-}$ blocs des algèbres de Hecke pour les groupes de réflexions exceptionnels spetsiaux, c'est à dire, pour les 18 groupes de réflexions irréductibles

$$
G_{4}, G_{6}, G_{8}, G_{14}, G_{23, \ldots, 30}, G_{32, \ldots, 37}
$$

dans la notation de Shephard-Todd (ceci couvre en particulier le cas du groupe de Coxeter de type $H_{4}$ qui ne pouvait être traité par les méthodes de [22]). Le cas des séries infinies a été traité par Broué et Kim [3]. Nous calculons aussi (partiellement) les matrices de décomposition pour chaque nombre premier mauvais.

1991 Mathematics Subject Classification. According to the 2000 classification : Primary 20C08; Secondary 20C40. 
Dans un second temps, nous démontrons (théorème 6.1) un résultat concernant les caractères unipotents de groupes réductifs finis : à l'intérieur d'une $d$-série de HarishChandra, les familles définies par l'algèbre de Hecke de la série sont les intersections des familles de Lusztig avec la $d$-série concernée. Ce résultat (établi cas par cas) peut être vu comme une généralisation des résultats de [22], qui ne concernent que le cas $d=1$ et la série principale.

Dans le cas particulier des 1-séries de Harish-Chandra, le théorème 6.1 apparaît comme une variante de la conjecture de Lusztig [16, (25.3)] sur les cellules bilatères dans les algèbres de Hecke usuelles avec des paramètres inégaux.

Les calculs ont été effectués à l'aide du logiciel Chevie 10, en utilisant en particulier les fonctions sur les groupes de réflexions complexes implémentées par Jean Michel. Nous lui adressons nos remerciements pour avoir fourni cet outil essentiel. Nous remercions aussi Jürgen Müller pour ses calculs de décomposition de représentations (en particulier, pour $G_{30}=W\left(H_{4}\right)$ ) avec les logiciels MeatAxe et VectorEnumerator.

\section{Algèbres de HeCke de Groupes de RÉFlexions COMPlexes et familles}

Nous commençons par rappeler la définition des algèbres de Hecke associées aux groupes de réflexions complexes et certaines de leurs propriétés. Ensuite, nous donnons quelques résultats qui seront utilisés plus tard pour la détermination des $\mathcal{O}$-blocs.

\subsection{Algèbres de Hecke.}

2.1.1. Soit $k$ un corps de nombres et $W$ un groupe de réflexions complexe fini sur le $k$-espace vectoriel $V$. Soit $\mathcal{H}=\mathcal{H}(W, x)$ l'algèbre de Hecke de $W$ sur l'anneau des polynômes de Laurent $A=\mathbb{Z}\left[x, x^{-1}\right]$.

Rappelons sa construction par Broué-Malle-Rouquier [8]. Soit $\mathcal{A}$ l'ensemble des hyperplans de réflexion de $W$ et $B_{W}$ le groupe de tresses de $W$, i.e., le groupe fondamental de

$$
X=\left(V-\bigcup_{H \in \mathcal{A}} H\right) / W
$$

relatif à un point base $x_{0}$. Alors, $\mathcal{H}$ est le quotient de l'algèbre de groupe $A\left[B_{W}\right]$ par l'idéal engendré par $\left(\sigma_{H}-x\right)\left(1+\sigma_{H}+\cdots+\sigma_{H}^{d-1}\right)$, où $H$ décrit $\mathcal{A}, d$ est l'ordre du fixateur de $H$ dans $W, s$ est la réflexion autour de $H$ de déterminant $\exp (2 i \pi / d)$ et $\sigma_{H}$ est un $s$-générateur de la monodromie autour de $H$.

La structure de ces algèbres de Hecke reste encore mystérieuse. Néanmoins on conjecture qu'elles partagent beaucoup des propriétés des algèbres d'Iwahori-Hecke usuelles.

2.1.2. Soit $W^{\prime}$ un sous-groupe parabolique de $W$. Dans [8, $\left.\S 4 . \mathrm{C}\right]$, on construit une injection de $\mathcal{H}\left(W^{\prime}\right)$ dans $\mathcal{H}$. Cette injection est définie à automorphisme intérieur de $\mathcal{H}$ près.

Conjecture 2.1. Pour tout sous-groupe parabolique $W^{\prime}$ de $W$, l'algèbre $\mathcal{H}$ est un module libre de rang $\left[W: W^{\prime}\right]$ sur $\mathcal{H}\left(W^{\prime}\right)$.

Notons que cette conjecture est indépendante du choix du plongement. On conjecture en particulier que $\mathcal{H}$ est un module libre de rang $|W|$ sur $A$.

On notera $A^{\prime} \mathcal{H}$ l'algèbre $A^{\prime} \otimes_{A} \mathcal{H}$, pour $A^{\prime}$ une $A$-algèbre commutative. 
Supposons dorénavant la conjecture 2.1 correcte. Alors, $\mathcal{H}$ est une déformation de $\mathbb{Z}[W]$ : on a un isomorphisme

$$
\mathcal{H} \otimes_{A}(A /(x-1)) \stackrel{\sim}{\longrightarrow} \mathbb{Z}[W]
$$

(ce morphisme est celui par lequel se factorise l'application canonique $\mathbb{Z}\left[B_{W}\right] \rightarrow \mathbb{Z}[W]$ provenant du revêtement galoisien $V-\bigcup_{H \in \mathcal{A}} H \rightarrow X$ ).

Soit $K$ une extension galoisienne finie de $k(x)$ telle que $K \mathcal{H}$ et $K[W]$ sont semisimples déployées (d'après Benard et Bessis [0], l'algèbre $k[W]$ est déployée et d'après Malle [20, Thm. 5.2], l'algèbre $\mathcal{H} \otimes_{A} k\left(x^{1 / \mu(k)}\right)$ est déployée, où $\mu(k)$ est l'ordre du groupe des racines de l'unité de $k$ ).

Soit $B$ la clôture intégrale de $A$ dans $K$ et $\mathfrak{n}$ un idéal premier de $B$ au-dessus de $(x-1)$. On déduit de (11) un isomorphisme $\left(B_{\mathfrak{n}} / \mathfrak{n}\right) \mathcal{H} \stackrel{\sim}{\longrightarrow}\left(B_{\mathfrak{n}} / \mathfrak{n}\right)[W]$. Puisque $\left(B_{\mathfrak{n}} / \mathfrak{n}\right) \mathcal{H}$ est semi-simple et déployée, l'application de décomposition est une bijection entre l'ensemble $\operatorname{Irr}(\mathcal{H})$ des caractères irréductibles de $K \mathcal{H}$ et l'ensemble des caractères irréductibles de $\left(B_{\mathfrak{n}} / \mathfrak{n}\right) \mathcal{H}$. De même, on a une bijection entre l'ensemble $\operatorname{Irr}(W)$ des caractères irréductibles de $K[W]$ et l'ensemble des caractères irréductibles de $\left(B_{\mathfrak{n}} / \mathfrak{n}\right)[W]$.

Par conséquent, on obtient une bijection

$$
\operatorname{Irr}(\mathcal{H}) \stackrel{\sim}{\longrightarrow} \operatorname{Irr}(W) .
$$

Dans la suite, nous identifierons les caractères irréductibles de $W$ et de $\mathcal{H}$ via cette bijection.

Cette bijection dépend du choix de l'idéal premier $\mathfrak{n}$. Les idéaux premiers au dessus de $(x-1)$ sont permutés par le groupe de Galois de $K / k(x)$. Soit maintenant $R$ une sous- $A$-algèbre de $K$ invariante par le groupe de Galois. La partition des caractères irréductibles de $K \mathcal{H}$ selon les blocs de $R \mathcal{H}$ est invariante par action du groupe de Galois : par conséquent, la partition correspondante de $\operatorname{Irr}(W)$ est indépendante du choix de $\mathfrak{n}$.

2.1.3. Dans Broué-Malle-Michel [7, Thm. 2.1], il est démontré qu'il existe au plus une forme symétrisante sur $\mathcal{H}$ vérifiant certaines propriétés : nous l'appellerons forme symétrisante canonique.

Conjecture 2.2. La forme symétrisante canonique sur $\mathcal{H}(W)$ existe et sa restriction à toute sous-algèbre parabolique $\mathcal{H}\left(W^{\prime}\right)$ est la forme symétrisante canonique.

Nous supposons aussi que la forme symétrisante construite dans 18 pour les groupes de réflexions complexes de rang 2 et dans [21] pour des groupes de rang supérieur est la forme canonique.

Rappelons qu'on ne sait pas, en général, que $\mathcal{H}$ est une algèbre symétrique.

Soit $t: \mathcal{H} \rightarrow A$ une forme symétrisante vérifiant la conjecture 2.2. Alors, on a une décomposition de l'extension de $t$ à $K$ :

$$
t=\sum_{\chi \in \operatorname{Irr}(W)} \frac{1}{c_{\chi}} \chi
$$

où nous avons identifié les caractères de $K \mathcal{H}$ et de $W$. Les éléments de Schur $c_{\chi}$ sont dans $B$ (cf. Geck-Rouquier [12, Prop. 4.2]). 
2.1.4. La conjecture suivante est vraie lorsque $W$ est un groupe de Weyl 『12, Thm. 5.2]. Le bon comportement du centre par extension des scalaires est une propriété importante pour l'étude des représentations d'une algèbre symétrique, car elle est équivalente à la propriété correspondante pour les fonctions de classe sur l'algèbre.

Conjecture 2.3. Soit $R$ une A-algèbre commutative locale de corps résiduel F. Alors, le morphisme canonique $Z(R \mathcal{H}) \rightarrow Z(F \mathcal{H})$ est surjectif.

2.2. Détermination des $\mathcal{O}$-blocs. Dans la suite, nous supposerons que $W$ vérifie les conjectures 2.1, 2.2 et 2.3.

Les conjectures 2.1 et 2.2 ont été vérifiées pour tous les groupes de réflexion complexes imprimitifs ainsi que pour des groupes primitifs d'ordre petit, via des méthodes informatiques. Quant à la conjecture 2.3, elle n'a pas été, à notre connaissance, vérifiée pour des groupes de réflexion non réels.

\subsubsection{Soit $\mathcal{O}=B\left[(1+x P(x))^{-1}\right]_{P \in \mathbb{Z}[x]}$.}

Nous identifierons comme à l'accoutumée les blocs d'une algèbre $C$ sur un anneau commutatif intègre $R$ avec l'ensemble des caractères irréductibles de $F \otimes_{R} C$, où $F$ est le corps des fractions de $R$.

Définition 2.4. Les familles de $\operatorname{Irr}(W)$ sont les blocs de $\mathcal{O H}(W)$ (via l'identification (2)).

D'après [22], cette définition coïncide avec la définition de Lusztig en termes de combinatoire de Kazhdan-Lusztig, pour les groupes de Weyl et plus généralement pour les groupes de Coxeter finis n'ayant pas de facteur de type $H_{4}$ (une propriété de positivité des constantes de structure de la base de Kazhdan-Lusztig est requise). Nos résultats montrent (cf. corollaire 3.2) que c'est en fait vrai pour tous les groupes de Coxeter finis.

On définit de manière inductive la notion de caractère constructible de $W$ : le caractère trivial du groupe trivial est constructible. Les caractères constructibles de $W$ sont les éléments de la famille génératrice minimale du sous-monoïde de $\mathbb{Z} \operatorname{Irr}(W)$ engendré par les $p_{\mathcal{F}}\left(\operatorname{Ind}_{W^{\prime}}^{W} \phi\right)$, où $W^{\prime}$ est un sous-groupe parabolique propre de $W, \phi$ un caractère constructible de $W^{\prime}, \mathcal{F}$ une famille de $\operatorname{Irr}(W)$ et $p_{\mathcal{F}}$ la projection sur $\mathbb{Z} \mathcal{F}$. Les caractères constructibles sont donc des caractères de $\mathcal{O H}$-modules projectifs.

Rappelons la construction des caractères constructibles au sens de Lusztig. C'est la donnée, pour chaque groupe de Coxeter fini, d'un ensemble minimal de caractères vérifiant les propriétés suivantes :

- le caractère trivial est constructible

- pour $\phi$ un caractère constructible d'un sous-groupe parabolique $W^{\prime}$ de $W$, alors le $J$-induit de $\phi$ est un caractère constructible de $W$

- le produit tensoriel par le caractère signe $\epsilon$ d'un caractère constructible est encore un caractère constructible.

Proposition 2.5. Lorsque $W$ est un groupe de Coxeter fini, un caractère est constructible (au sens défini ci-dessus) si et seulement si il est constructible au sens de Lusztig.

Preuve. Le caractère trivial est seul dans sa famille et le caractère signe $\epsilon$ est constructible. La $J$-induction est une induction suivie par une projection sur une union 
de familles. Par conséquent, un caractère constructible au sens de Lusztig est une somme de caractères constructibles.

Il suffit de démontrer la proposition pour $W$ irréductible, ce que nous supposons maintenant.

Si $W$ n'est pas de type $F_{4}$ ou $E_{n}$, alors les caractères constructibles au sens de Lusztig forment une base du sous-monoïde des caractères formé des caractères des $\mathcal{O H}$-modules projectifs [22, Thm. 2]. Or, les caractères constructibles sont des caractères de $\mathcal{O H}$-modules projectifs. Par conséquent, les caractères constructibles sont les caractères constructibles au sens de Lusztig.

Le cas où $W$ est de type $F_{4}$ ou $E_{n}$ se vérifie directement par calcul de tous les induits de caractères constructibles de sous-groupes paraboliques stricts maximaux.

2.2.2. Soit $p$ un nombre premier, $\mathfrak{p}$ un idéal premier de $\mathcal{O}$ au-dessus de $p$ et $k_{\mathfrak{p}}$ le corps des fractions de $\mathcal{O}_{\mathfrak{p}} / \mathfrak{p}$.

Nous renvoyons le lecteur à Geck-Rouquier [12, §2] pour le traitement des applications de décomposition (extension au cas d'un anneau de base intégralement clos des résultats classiques pour un anneau de valuation discrète).

On dispose d'une application de décomposition du groupe de Grothendieck $G_{0}(K \mathcal{H})$ de la catégorie des $K \mathcal{H}$-modules de type fini vers $G_{0}\left(k_{\mathfrak{p}} \mathcal{H}\right)$ et d'une application de Car$\tan c$ du groupe de Grothendieck $K_{0}\left(k_{\mathfrak{p}} \mathcal{H}\right)$ de la catégorie des $k_{\mathfrak{p}} \mathcal{H}$-modules projectifs de type fini vers $G_{0}(K \mathcal{H})$ qui est duale de l'application de décomposition. L'application de Cartan est injective (on utilise ici la conjecture 2.3) [12, Prop. 3.1]. Nous appellerons caractère virtuellement projectif $\left(\right.$ de $\left.\mathcal{O}_{\mathfrak{p}} \mathcal{H}\right)$ un élément de $G_{0}(K \mathcal{H})$ dans l'image de l'application de Cartan. Lorsque cet élément provient d'un $k_{\mathfrak{p}} \mathcal{H}$-module projectif, nous dirons que c'est un caractère projectif.

Les familles de caractères de $W$ sont les sous-ensembles minimaux de $\operatorname{Irr}(W)$ qui sont réunion de blocs de $\mathcal{O}_{\mathfrak{p}} \mathcal{H}$ pour tout nombre premier $p$.

Rappelons quelques propriétés classiques utilisant la structure symétrique de $\mathcal{O}_{\mathfrak{p}} \mathcal{H}$.

Lemme 2.6. (a) Soit $\phi$ un caractère virtuellement projectif de $\mathcal{O}_{\mathfrak{p}} \mathcal{H}$. Alors,

$$
\sum_{\chi \in \operatorname{Irr}(W)} \frac{\langle\phi, \chi\rangle}{c_{\chi}} \in \mathcal{O}_{\mathfrak{p}} .
$$

(b) $\chi$ est seul dans son bloc $\Longleftrightarrow c_{\chi} \notin \mathfrak{p} \Longleftrightarrow \chi$ est un caractère projectif.

(c) Si deux caractères irréductibles $\chi$ et $\psi$ sont dans le même bloc de $\mathcal{O}_{\mathfrak{p}} \mathcal{H}$, alors ils sont dans le même bloc de $\mathcal{O}_{\mathfrak{p}}[W]$. Tout caractère projectif de $\mathcal{O}_{\mathfrak{p}}[W]$ est un caractère projectif de $\mathcal{O}_{\mathfrak{p}} \mathcal{H}$.

(d) Soit $W^{\prime}$ un sous-groupe parabolique de $W$. Alors, la restriction d'un caractère projectif de $\mathcal{O}_{\mathfrak{p}} \mathcal{H}$ à $\mathcal{O}_{\mathfrak{p}} \mathcal{H}\left(W^{\prime}\right)$ est un caractère projectif. De même, l'induit d'un caractère projectif de $\mathcal{O}_{\mathfrak{p}} \mathcal{H}\left(W^{\prime}\right)$ est un caractère projectif de $\mathcal{O}_{\mathfrak{p}} \mathcal{H}$.

Preuve. L'assertion (a) est [12, Prop. 4.4].

Si $\chi$ est un caractère projectif, il résulte de (a) que $c_{\chi} \notin \mathfrak{p}$.

Soit $C$ le bloc de $\mathcal{O}_{\mathfrak{p}} \mathcal{H}$ contenant $\chi$. Si $c_{\chi}$ est inversible dans $\mathcal{O}_{\mathfrak{p}}$, alors l'idempotent central $e_{\chi}$ associé à $\chi$ est dans $\mathcal{O}_{\mathfrak{p}} \mathcal{H}$, donc $\chi$ est seul dans son bloc, $k_{\mathfrak{p}} C$ est une algèbre simple et $\chi$ est un caractère projectif. 
Si $\chi$ est seul dans son bloc, alors $Z(C)$ est de rang 1 sur $\mathcal{O}_{\mathfrak{p}}$, donc $Z\left(k_{\mathfrak{p}} C\right)$ est de dimension 1 (conjecture 2.3). Par conséquent, le socle de $k_{\mathfrak{p}} C$ est nul, donc $k_{\mathfrak{p}} C$ est une algèbre simple et $\chi$ est un caractère projectif.

Si $\chi$ est un caractère projectif, alors $\chi$ est seul dans son bloc. Ceci achève la preuve de (b).

Soit $\mathfrak{q}$ un idéal premier de $B$ contenant $\mathfrak{p}$ et $\mathfrak{n}(\mathfrak{n}$ est un idéal premier de $B$ au-dessus de $(x-1))$. La surjection canonique

$$
\left(B_{\mathfrak{q}}\right) \mathcal{H} \rightarrow\left(B_{\mathfrak{q}} / \mathfrak{n}\right) \mathcal{H} \stackrel{\sim}{\longrightarrow}\left(B_{\mathfrak{q}} / \mathfrak{n}\right)[W]
$$

envoie un bloc sur un bloc. Puisqu'un bloc de $\left(B_{\mathfrak{q}}\right) \mathcal{H}$ est une somme de blocs de $\left(B_{\mathfrak{p}}\right) \mathcal{H}=\mathcal{O}_{\mathfrak{p}} \mathcal{H}$ et que les blocs de $\left(B_{\mathfrak{q}} / \mathfrak{n}\right)[W]$ coïncident avec ceux de $\mathcal{O}_{\mathfrak{p}}[W]$, on obtient la première partie de (c). La seconde partie de (c) résulte de la transitivité des applications de décomposition [12, Prop. 2.12].

Puisque $\mathcal{O}_{\mathfrak{p}} \mathcal{H}$ est un $\mathcal{O}_{\mathfrak{p}} \mathcal{H}\left(W^{\prime}\right)$-module projectif par conjecture 2.1, les foncteurs d'induction et de restriction entre ces algèbres sont exacts, d'où $(d)$.

Soit $W^{\prime}$ un sous-groupe parabolique de $W$. On considère le morphisme canonique

$$
\mathcal{H}(W) \rightarrow \mathcal{H}(W) \otimes_{\mathcal{H}\left(W^{\prime}\right)} \mathcal{H}(W) \rightarrow \mathcal{H}(W)
$$

composition de l'unité et de la counité provenant de la paire biadjointe $\left(\operatorname{Res}_{\mathcal{H}\left(W^{\prime}\right)}^{\mathcal{H}(W)}, \operatorname{Ind}_{\mathcal{H}\left(W^{\prime}\right)}^{\mathcal{H}(W)}\right)$. On note $\operatorname{Tr}_{\mathcal{H}\left(W^{\prime}\right)}^{\mathcal{H}(W)} 1 \in Z(\mathcal{H})$ l'image de 1 par ce morphisme. Alors, on a le lemme classique suivant :

Lemme 2.7. L'action de $\operatorname{Tr}_{\mathcal{H}\left(W^{\prime}\right)}^{\mathcal{H}(W)} 1$ sur un $K \mathcal{H}$-module simple de caractère $\chi$ est donnée par l'élément de $\mathcal{O}$

$$
c_{\chi} \sum_{\psi \in \operatorname{Irr}\left(W^{\prime}\right)} \frac{\left\langle\operatorname{Res}_{W^{\prime}}^{W} \chi, \psi\right\rangle}{c_{\psi}} .
$$

Soit $B$ un bloc de $\mathcal{O}_{\mathfrak{p}} \mathcal{H}(W)$. Alors, l'image dans $k_{\mathfrak{p}}$ du scalaire par lequel $\operatorname{Tr}_{\mathcal{H}\left(W W^{\prime}\right)}^{\mathcal{H}(W)} 1$ agit sur un KB-module simple est indépendante du module simple. En outre, $B$ est projectif relativement à $\mathcal{O}_{\mathfrak{p}} \mathcal{H}\left(W^{\prime}\right)$ si et seulement si ce scalaire est inversible.

Preuve. Pour la formule, voir par exemple [11, Lemma 9.4.6].

Pour le reste, on utilise la propriété de $Z\left(k_{\mathfrak{p}} B\right)$ d'être local. Un élément de $Z(B)$ est inversible si et seulement si son image dans le corps résiduel de $Z\left(k_{\mathfrak{p}} B\right)$ est non nulle, ce qui explique la dernière assertion.

Soit $S(V)$ l'algèbre symétrique de l'espace vectoriel $V$. Alors $S(V)$ est un $k[W]$ module gradué. L'algèbre des coinvariants de $W$ est le quotient de $S(V)$ par l'idéal engendré par les invariants de degré positif, $R=S(V) /\left(S(V)_{+}^{W}\right)$. C'est encore un $k[W]$-module gradué. Le polynôme de Poincaré $P(W) \in \mathbb{Z}[x]$ de $W$ est la dimension graduée de $R$. Pour $\chi \in \operatorname{Irr}(W)$ le degré fantôme $R_{\chi} \in \mathbb{Z}[x]$ est défini comme la multiplicité graduée de $\chi$ dans le caractère de $R$. Notons $N(\chi)=\left.\frac{d R_{\chi}}{d x}\right|_{x=1}$. Pour $\chi \in \operatorname{Irr}(W)$, nous notons $\chi^{*}$ le caractère conjugué.

Le résultat suivant est utile pour la détermination des blocs : 
Lemme 2.8. L'application

$$
\operatorname{Irr}(W) \longrightarrow \mathbb{Z}, \quad \chi \mapsto\left(N(\chi)+N\left(\chi^{*}\right)\right) / \chi(1),
$$

est constante sur les blocs de $\mathcal{O H}$.

Preuve. D'après Broué-Michel [9, Prop. 4.18], on a

$$
\chi\left(T_{\boldsymbol{\pi}}\right)=\chi(1) x^{N+N^{*}-\left(N(\chi)+N\left(\chi^{*}\right)\right) / \chi(1)},
$$

où $\boldsymbol{\pi}$ est la classe du lacet $t \mapsto \exp (2 i \pi t) x_{0}$ (un élément du centre de $B_{W}$ ), $T_{\boldsymbol{\pi}}$ son image dans $\mathcal{H}$ (voir aussi Broué-Malle-Michel [7, Prop. 6.7(3)]), $N$ est le nombre d'hyperplans de réflexion de $W$ et $N^{*}$ le nombre de réflexions. On déduit alors le lemme de la propriété de la restriction de $\frac{1}{\chi(1)} \chi$ modulo $\mathfrak{p}$ au centre d'un bloc d'être indépendante du caractère irréductible $\chi$ du bloc.

2.3. Groupes spetsiaux. Suivant [21] nous dirons que le groupe de réflexions complexe $W$ est spetsial si tous les éléments de Schur $c_{\chi}$ de $\mathcal{H}(W)$ sont dans $A$. Les groupes de réflexions spetsiaux sont déterminés dans [21, 8A]. Notre but est la détermination des familles de $W$ et des matrices de décomposition de $\mathcal{O}_{\mathfrak{p}} \mathcal{H}$. D'après le lemme 2.6(b), l'application de décomposition est l'identité si $c_{\chi} \notin \mathfrak{p}$ pour tout $\chi$. Nous dirons que $p$ est un nombre premier mauvais pour $W$ si $p$ divise un des $c_{\chi}$, pour un $\chi \in \operatorname{Irr}(W)$. Il nous suffit donc de considérer les matrices de décomposition pour des idéaux $\mathfrak{p}$ au-dessus des mauvais nombres premiers.

Dans la table 1 nous indiquons les mauvais nombres premiers pour les groupes de réflexions spetsiaux exceptionnels (i.e., pour les groupes qui ne font pas partie des séries infinies).

TAB. 1. Mauvais nombres premiers

\begin{tabular}{|l|c|l|l|c|}
\hline \multicolumn{1}{|c|}{$W$} & mauvais $p$ & & \multicolumn{1}{|c|}{$W$} & mauvais $p$ \\
\hline$G_{4}$ & 2,3 & $G_{28}=W\left(F_{4}\right)$ & 2,3 \\
$G_{6}$ & 2,3 & $G_{29}$ & 2,5 \\
$G_{8}$ & 2,3 & $G_{30}=W\left(H_{4}\right)$ & $2,3,5$ \\
$G_{14}$ & 2,3 & $G_{32}$ & $2,3,5$ \\
$G_{23}=W\left(H_{3}\right)$ & 2,5 & $G_{33}$ & 2,3 \\
$G_{24}$ & 2,7 & $G_{34}$ & $2,3,7$ \\
$G_{25}$ & 2,3 & $G_{35}=W\left(E_{6}\right)$ & 2,3 \\
$G_{26}$ & 2,3 & $G_{36}=W\left(E_{7}\right)$ & 2,3 \\
$G_{27}$ & $2,3,5$ & $G_{37}=W\left(E_{8}\right)$ & $2,3,5$ \\
\hline
\end{tabular}

\section{Description Des familles et Des MATRICES DE DÉCOMPOSITION}

3.1. Démarche algorithmique. Nous allons donner les matrices de décomposition de $\mathcal{O}_{\mathfrak{p}} \mathcal{H}(W)$ pour tous les groupes de réflexions complexes spetsiaux irréductibles $W$, à des indéterminations près pour $W=G_{29}, p=2, W=G_{32}, p=2,3$ et $W=G_{34}$, $p=3$. Le cas des groupes de Weyl $F_{4}, E_{6}$ et $E_{7}$ a été résolu par Gyoja [13].

La notation utilisée pour les caractères coïncide avec celle du système Chevie [10].

Commençons par décrire notre démarche algorithmique. 
Nous procédons par induction. Soit $W$ un groupe de réflexions complexe et $p$ un nombre premier mauvais. Les $p$-blocs de $W$ se déterminent immédiatement à partir de la table de caractères de $W$. Supposons que les degrés fantômes $R_{\chi}$ et les éléments de Schur $c_{\chi}$ sont connus ainsi que les matrices de décomposition correspondantes pour les algèbres de Hecke de tous les sous-groupes paraboliques stricts (maximaux) de $W$. Alors une approximation de la matrice de décomposition est construite par la méthode suivante :

(1) Une partition de $\operatorname{Irr}(W)$ en union de blocs est donnée par l'intersection des $p$ blocs de $W$ avec les sous-ensembles sur lesquels $\left(N(\chi)+N\left(\chi^{*}\right)\right) / \chi(1)$ est constant (cf. lemmes 2.8 et 2.6(c)). De plus, chaque $\chi$ avec $c_{\chi} \notin \mathfrak{p}$ forme un bloc en luimême (cf. lemme 2.6(b)).

(2) Une approximation des caractères projectifs indécomposables s'obtient comme suit. Soit $P$ l'ensemble des induits des caractères projectifs indécomposables des sous-algèbres paraboliques maximales, coupés par les approximations des blocs obtenus à la première étape. On construit alors la famille $U$ génératrice du sousmonoïde de $\mathbb{Z} \operatorname{Irr}(W)$ engendré par $P$.

(3) On définit une relation d'équivalence comme clôture transitive de la relation suivante $\operatorname{sur} \operatorname{Irr}(W)$ : deux caractères $\chi_{1}, \chi_{2}$ sont reliés si ils sont dans une même partie de la partition construite en (1) et si de plus il existe un caractère $\phi \in U$ tel que $\chi_{1}$ et $\chi_{2}$ interviennent dans $\phi$. On obtient ainsi un raffinement de la partition construite en (1) où les parties sont toujours des unions de blocs.

(4) Par construction, les éléments de $U$ sont des caractères projectifs. Supposons que $\phi \in U$ n'est pas indécomposable. Alors, il existe des caractères non nuls $\phi_{1}$ et $\phi_{2}$ avec $\phi_{1}+\phi_{2}=\phi$ et $\sum_{\chi}\left\langle\phi_{1}, \chi\right\rangle / c_{\chi} \in \mathcal{O}_{\mathfrak{p}}$ (cf. lemme 2.6(a)). Par conséquent, si la somme précedente n'est contenue dans $\mathcal{O}_{\mathfrak{p}}$ pour aucun sous-caractère propre de $\phi$, alors $\phi$ est indécomposable.

Les points (1)-(4) suffisent pour obtenir les matrices de décomposition pour tous les mauvais nombres premiers lorsque $W=G_{i}$ avec $i \in\{4,6,23,26,27,33\}$. Pour les autres groupes, nous aurons besoin d'arguments supplémentaires.

Les étapes décrites ci-dessus peuvent toutes être effectuées manuellement. Néanmoins, nous avons utilisé la librairie Chevie pour organiser et manipuler les tables plus aisément et pour réduire les risques d'erreurs. Par exemple, pour $W=G_{24}$, les instructions suivantes ont été utilisées :

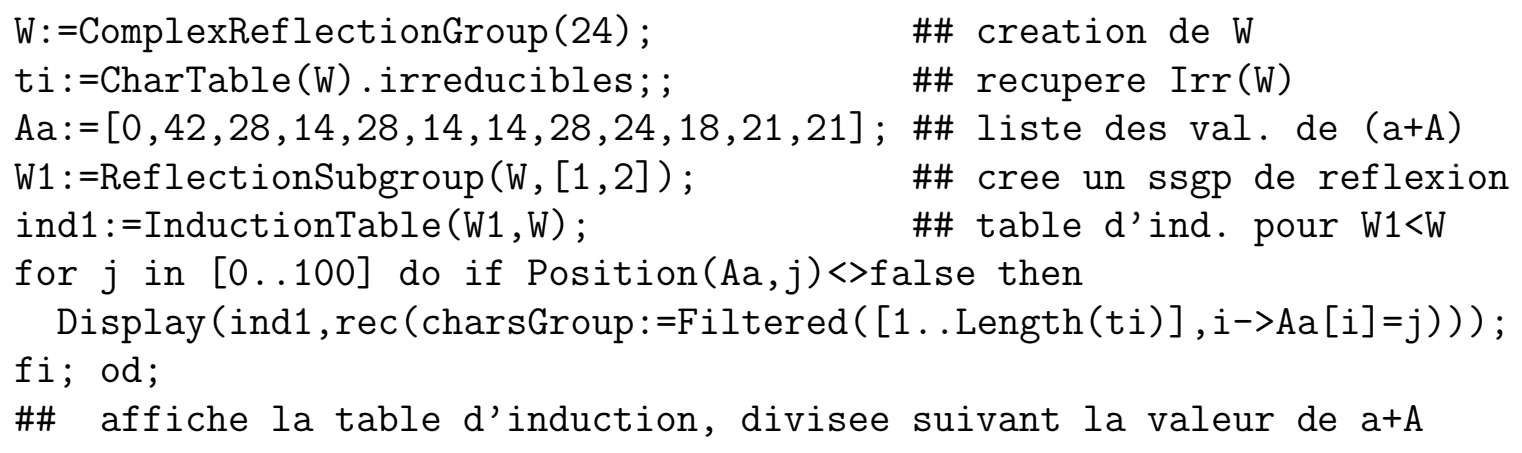

Pour utiliser l'information sur les $p$-blocs de $W$, les instructions GAP suivantes

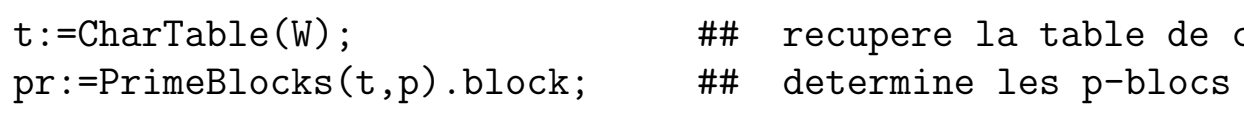


3.2. Types de familles. De nombreux blocs de $\mathcal{O H}(W)$ présentent des matrices de décomposition identiques pour des groupes $W$ différents. Aussi, nous introduisons ici les types rencontrés plus d'une fois. Nous indiquons la matrice de décomposition pour chaque nombre premier $p$ où elle n'est pas l'identité. Nous indiquons aussi le plus petit coefficient non nul $f_{\chi}$ de $c_{\chi}$ et dans la colonne $c$, les caractères constructibles.

\begin{tabular}{c|c|c|c|} 
& $f_{\chi}$ & $p$ & $c$ \\
\hline$\chi_{1}$ & $\frac{1}{2} p^{n}\left(1+\sqrt{1-4 p^{-n}}\right)$ & 1 & 1 \\
$\chi_{2}$ & $\frac{1}{2} p^{n}\left(1-\sqrt{1-4 p^{-n}}\right)$ & 1 & 1 \\
\hline
\end{tabular}

Type $\alpha_{1^{2}}\left(p^{n}\right)$

\begin{tabular}{l|c|l|l|} 
& $f_{\chi}$ & $p=2$ & $c$ \\
\hline$\chi_{1}$ & $2(1-\sqrt{-1})$ & 1 & 1 \\
$\chi_{2}$ & 2 & 1 & 1 \\
$\chi_{3}$ & $2(1+\sqrt{-1})$ & 1 & 1 \\
\hline
\end{tabular}

Type $\alpha_{1^{3}}(2)$

\begin{tabular}{l|l|l|l|} 
& $f_{\chi}$ & $p=2$ & $c$ \\
\hline$\chi_{1}$ & 4 & 1 & 1 \\
$\chi_{2}$ & 2 & 1 & 1 \\
$\chi_{3}$ & 4 & 1 & 1 \\
\hline
\end{tabular}

Type $\beta_{1^{3}}(2)$

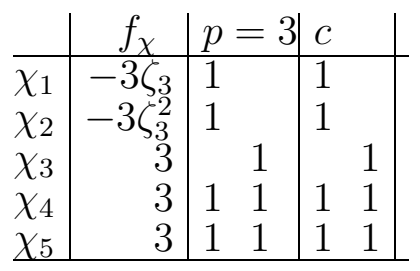

Type $\mathcal{M}\left(Z_{3}\right)$

\begin{tabular}{l|l|l|l|} 
& $f_{\chi}$ & $p=3$ & $c$ \\
\hline$\chi_{1}$ & 3 & 1 & 1 \\
$\chi_{2}$ & 3 & 1 & 1 \\
$\chi_{3}$ & 3 & 1 & 1 \\
\hline
\end{tabular}

Type $\alpha_{1^{3}}(3)$

\begin{tabular}{l|r|l|l|} 
& \multicolumn{1}{|c|}{$f_{\chi}$} & $p=3$ & $c$ \\
\hline$\chi_{1}$ & $-3 \zeta_{3}$ & 1 & 1 \\
$\chi_{2}$ & 3 & 1 & 1 \\
$\chi_{3}$ & 3 & 1 & 1 \\
$\chi_{4}$ & $-3 \zeta_{3}^{2}$ & 1 & 1 \\
\hline
\end{tabular}

Type $\alpha_{1^{4}}(3)$

\begin{tabular}{l|l|ll|ll|} 
& $f_{\chi}$ & $p=2$ & $c$ & \\
\hline$\chi_{1}$ & 2 & 1 & & 1 & \\
$\chi_{2}$ & 2 & & 1 & & 1 \\
$\chi_{3}$ & 2 & 1 & 1 & 1 & 1 \\
\hline
\end{tabular}

Type $\mathcal{M}\left(Z_{2}\right)$

\begin{tabular}{l|r|rr|ll|} 
& $f_{\chi}$ & $p=3$ & $c$ & \\
\hline$\chi_{1}$ & $3 \zeta_{3}$ & 1 & & 1 & \\
$\chi_{2}$ & $3 \zeta_{3}^{2}$ & 1 & & 1 & \\
$\chi_{3}$ & 3 & & 1 & 1 & 1 \\
$\chi_{4}$ & 3 & & 1 & 1 & 1 \\
$\chi_{5}$ & 3 & 1 & 1 & 2 & 1 \\
\hline
\end{tabular}

Type $\mathcal{M}\left(Z_{3}\right)^{\prime}$

\begin{tabular}{l|r|rr|rr|} 
& $f_{\chi}$ & $p=3$ & $c$ & \\
\hline$\chi_{1}$ & 3 & 1 & & 1 & \\
$\chi_{2}$ & 3 & 1 & & 1 & \\
$\chi_{3}$ & 3 & & 1 & & 1 \\
$\chi_{4}$ & 3 & & 1 & & 1 \\
$\chi_{5}$ & 3 & 1 & 1 & 1 & 1 \\
\hline
\end{tabular}

Type $\mathcal{M}\left(Z_{3}\right)^{\prime \prime}$

\begin{tabular}{|c|c|c|c|c|}
\hline & $f_{\chi}$ & $p=2$ & $p=r$ & $c$ \\
\hline$\chi_{1}$ & $r+\sqrt{4 r-15}$ & 1 & 1 & 1 \\
\hline$\chi_{2}$ & $r+\sqrt{4 r-15}$ & & 1 & 1 \\
\hline$x_{3}$ & $r-\sqrt{4 r-15}$ & 1 & 1 & 1 \\
\hline & $r-\sqrt{4 r-15}$ & 1 & 1 & 1 \\
\hline
\end{tabular}

Type $\alpha_{3 \times 4}(r), r \neq 2$

\begin{tabular}{|c|c|c|c|c|}
\hline & $f_{\chi}$ & $p=2$ & $p=r$ & $c$ \\
\hline$\chi_{1}$ & $-2 \sqrt{-r}$ & 1 & 1 & 1 \\
\hline$\chi_{2}$ & $2 \sqrt{-r}$ & 1 & 1 & 1 \\
\hline$\chi_{3}$ & 2 & 11 & 1 & 2 \\
\hline
\end{tabular}

Type $\alpha_{G_{4}}(r), r \neq 2$

\begin{tabular}{|c|c|c|c|}
\hline$f_{\chi}$ & $p=2$ & $p=3$ & $c$ \\
\hline 3 & 1 & 1 & 1 \\
\hline 6 & 1 & 1 & 1 \\
\hline 2 & 1 & 1 & $\begin{array}{lll}111 & 1\end{array}$ \\
\hline 6 & 11 & 1 & $\begin{array}{ll}1 & 1\end{array}$ \\
\hline 3 & 1 & $\begin{array}{ll}1 & 1 \\
\end{array}$ & 11 \\
\hline
\end{tabular}

Type $\mathcal{M}\left(S_{3}\right)$ 


\begin{tabular}{|c|c|c|c|c|}
\hline & $f_{\chi}$ & $p=2$ & $p=3$ & $c$ \\
\hline$\chi_{1}$ & $6 \zeta_{3}^{2}$ & 1 & 1 & 1 \\
\hline$\chi_{2}$ & $6 \zeta_{3}$ & 1 & 1 & 1 \\
\hline$\chi_{3}$ & & 1 & 1 & \\
\hline$\chi_{4}$ & $-6 \zeta_{3}$ & 1 & 11 & 1 \\
\hline$\chi_{5}$ & $-6 \zeta_{3}^{2}$ & 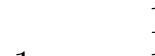 & 11 & 1 \\
\hline$\chi_{6}$ & $3-\sqrt{-3}$ & 1 & 1 & 2 \\
\hline$\chi_{7}$ & $3+\sqrt{-3}$ & 1 & 1 & 2 \\
\hline
\end{tabular}

Type $\alpha_{3 \times 5}$

\begin{tabular}{|c|c|c|c|c|}
\hline & $f_{\chi}$ & $p=2$ & $p=3$ & $c$ \\
\hline$\langle 1$ & $6 \zeta_{3}^{2}$ & 1 & 1 & 1 \\
\hline$\chi_{2}$ & $6 \zeta_{3}$ & 1 & 1 & 1 \\
\hline$\chi_{3}$ & $-6 \zeta_{3}$ & 1 & 1 & 1 \\
\hline$\chi_{4}$ & $-6 \zeta_{3}^{2}$ & 1 & 1 & 1 \\
\hline$\chi_{5}$ & $3-\sqrt{-3}$ & 1 & 1 & 2 \\
\hline$\underline{\chi_{6}}$ & $3+\sqrt{-3}$ & 11 & 1 & 2 \\
\hline
\end{tabular}

Type $\alpha_{3 \times 5}^{\prime}$

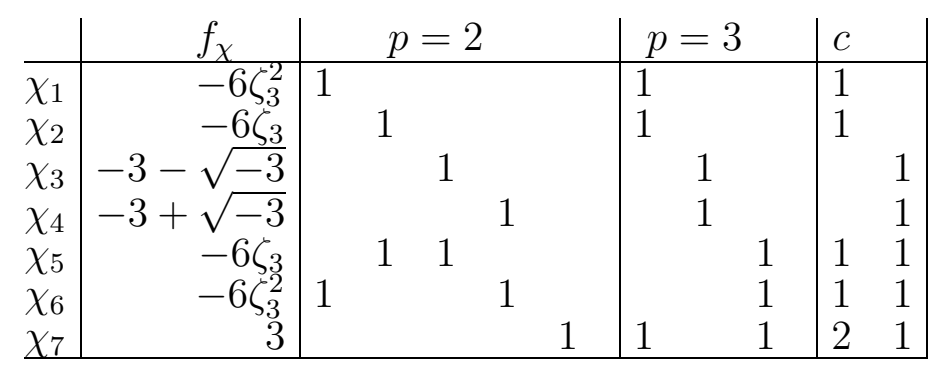

\section{Type $\alpha_{3 \times 5}^{\prime \prime}$}

Dans la table 2, nous indiquons les types de familles qui apparaissent dans un groupe de réflexions spetsial exceptionnel donné.

3.3. $W=G_{4}$. Soit $W=G_{4}$ le groupe primitif de réflexions complexe de dimension 2 et d'ordre 24. Pour $\chi \in \operatorname{Irr}(W)$, on pose $d(\chi)=\chi(1)$ et on note $b(\chi)$ la valuation en $x$ du degré fantôme $R_{\chi}$. Les caractères irréductibles $\chi$ sont uniquement déterminés par les paires $(d(\chi), b(\chi))$ associées et nous notons $\chi=\phi_{d, b}$ lorsque $d=d(\chi)$ et $b=b(\chi)$.

Le groupe de réflexions $G_{4}$ a 4 familles de caractères, des types suivants :

- défaut $0:\left(\phi_{1,0}\right),\left(\phi_{3,2}\right)$

- type $\alpha_{1^{2}}(3)$ : $\left(\phi_{2,1}, \phi_{2,3}\right)$

- type $\alpha_{G_{4}}(3):\left(\phi_{1,4}, \phi_{1,8}, \phi_{2,5}\right)$

3.4. $W=G_{6}$. Les caractères irréductibles de $G_{6}$ ne sont pas distingués par leur degré et leur invariant $b$. Dans les cas ambigus, nous donnons en plus comme troisième indice le degré en $x$ du degré fantôme $R_{\chi}$.

Le groupe de réflexions $G_{6}$ a 5 familles de caractères, des types suivants :

- défaut $0:\left(\phi_{1,0}\right),\left(\phi_{3,4}\right)$

- type $\alpha_{1^{2}}\left(2^{2}\right):\left(\phi_{2,5,13}, \phi_{2,7}\right)$

- type $\alpha_{1^{2}}(3):\left(\phi_{1,10}, \phi_{1,14}\right)$

- Autre famille : 
TAB. 2. Types de familles

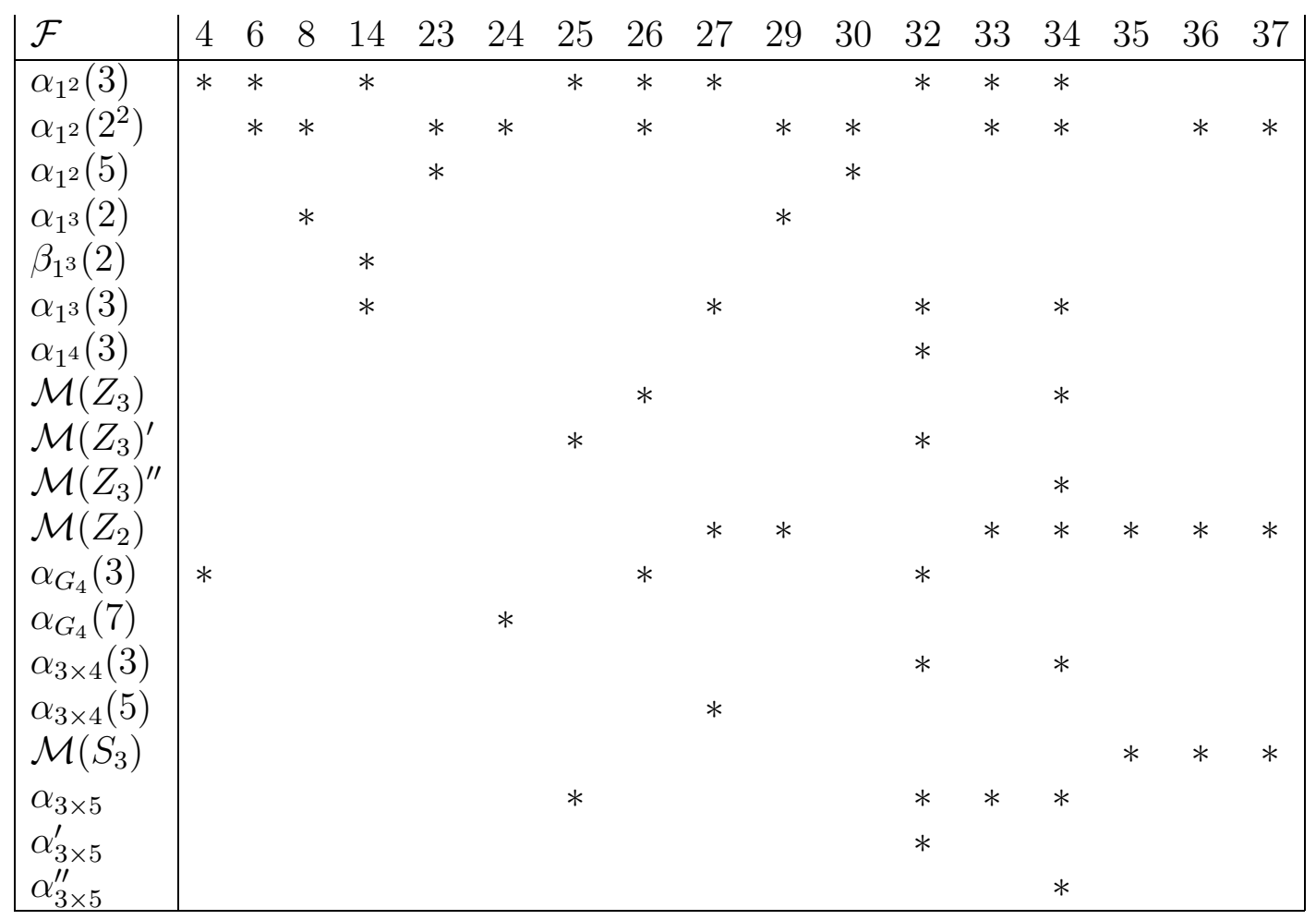

\begin{tabular}{|c|c|c|c|c|c|c|c|c|c|}
\hline & $f_{\chi}$ & \multicolumn{3}{|c|}{$p=2$} & \multicolumn{3}{|c|}{$p=3$} & \multicolumn{2}{|l|}{$c$} \\
\hline$\overline{\phi_{1,4}}$ & $-4 \sqrt{-3}$ & 1 & & & 1 & & & 1 & \\
\hline$\phi_{1,8}$ & $4 \sqrt{-3}$ & & 1 & & 1 & & & 1 & \\
\hline$\phi_{1,6}$ & & & & 1 & & 1 & & & 1 \\
\hline$\phi_{2,1}$ & $2(1+\sqrt{-1})(3+\sqrt{3})$ & 1 & & 1 & & 1 & & 1 & 1 \\
\hline$\phi_{2,5,9}$ & $2(1+\sqrt{-1})(3-\sqrt{3})$ & & 1 & 1 & & 1 & & 1 & 1 \\
\hline$\phi_{2,3,7}$ & $2(1-\sqrt{-1})(3-\sqrt{3})$ & 1 & & 1 & & & 1 & 1 & 1 \\
\hline$\phi_{2,3,11}$ & $2(1-\sqrt{-1})(3+\sqrt{3})$ & & 1 & 1 & & & 1 & 1 & 1 \\
\hline$\phi_{32}$ & 4 & 1 & 1 & 1 & & & 1 & 2 & 1 \\
\hline
\end{tabular}

3.5. $W=G_{8}$. Le groupe de réflexions complexe $G_{8}$ possède une paire de caractères irréductibles de mêmes degré et invariant $b$. Nous noterons $\phi_{2,7}^{\prime}$ le conjugué complexe de $\phi_{2,1}$, et $\phi_{2,7}^{\prime \prime}$ le conjugué de $\phi_{2,13}$.

Le groupe de réflexions $G_{8}$ a 5 familles de caractères, des types suivants :

- défaut $0:\left(\phi_{1,0}\right)$

- type $\alpha_{1^{2}}\left(2^{2}\right):\left(\phi_{4,3}, \phi_{4,5}\right)$

- type $\alpha_{1^{3}}(2):\left(\phi_{2,1}, \phi_{2,4}, \phi_{2,7}^{\prime}\right),\left(\phi_{3,2}, \phi_{3,4}, \phi_{3,6}\right)$

- Autre famille : 


\begin{tabular}{|c|c|c|c|c|c|c|c|}
\hline & $f_{\chi}$ & $p=2$ & & $p=3$ & & & $c$ \\
\hline$\overline{\phi_{1,6}}$ & -12 & 1 & 1 & & & & 1 \\
\hline$\phi_{1,18}$ & -12 & 1 & & 1 & & & 1 \\
\hline$\phi_{1,12}$ & 4 & 1 & & 1 & & & 1 \\
\hline$\phi_{2,10}$ & 12 & 2 & 1 & 1 & & & 2 \\
\hline$\phi_{2,7}^{\prime \prime}$ & $-4 \sqrt{-1}$ & 2 & & & 1 & & 2 \\
\hline$\phi_{2,13}$ & $4 \sqrt{-1}$ & 2 & & & 1 & & 2 \\
\hline$\phi_{3,8}$ & 4 & 3 & & & & 1 & 3 \\
\hline
\end{tabular}

Le seul caractère projectif pour $p=2$ dans la famille à 7 éléments est indécomposable, car tous les caractères de cette famille se factorisent (modulo $\mathfrak{p}$ ) par l'algèbre de groupe $k_{\mathfrak{p}}[W]$. Pour $W$, le caractère projectif correspondant est indécomposable.

3.6. $W=G_{14}$. Les caractères irréductibles de $G_{14}$ ne sont pas distingués par leur degré et leur invariant $b$. Dans les cas ambigus, nous donnons en plus comme troisième indice le degré en $x$ du degré fantôme $R_{\chi}$.

Le groupe de réflexions $G_{14}$ a 6 familles de caractères, des types suivants :

- défaut $0:\left(\phi_{1,0}\right)$

- type $\beta_{1^{3}}(2):\left(\phi_{2,9}, \phi_{2,12}, \phi_{2,15}\right)$

- type $\alpha_{1^{2}}(3):\left(\phi_{4,5}, \phi_{4,7}\right),\left(\phi_{1,20}, \phi_{1,28}\right)$

- type $\alpha_{1^{3}}(3):\left(\phi_{3,8}, \phi_{3,6,24}, \phi_{3,10}\right)$

- Autre famille :

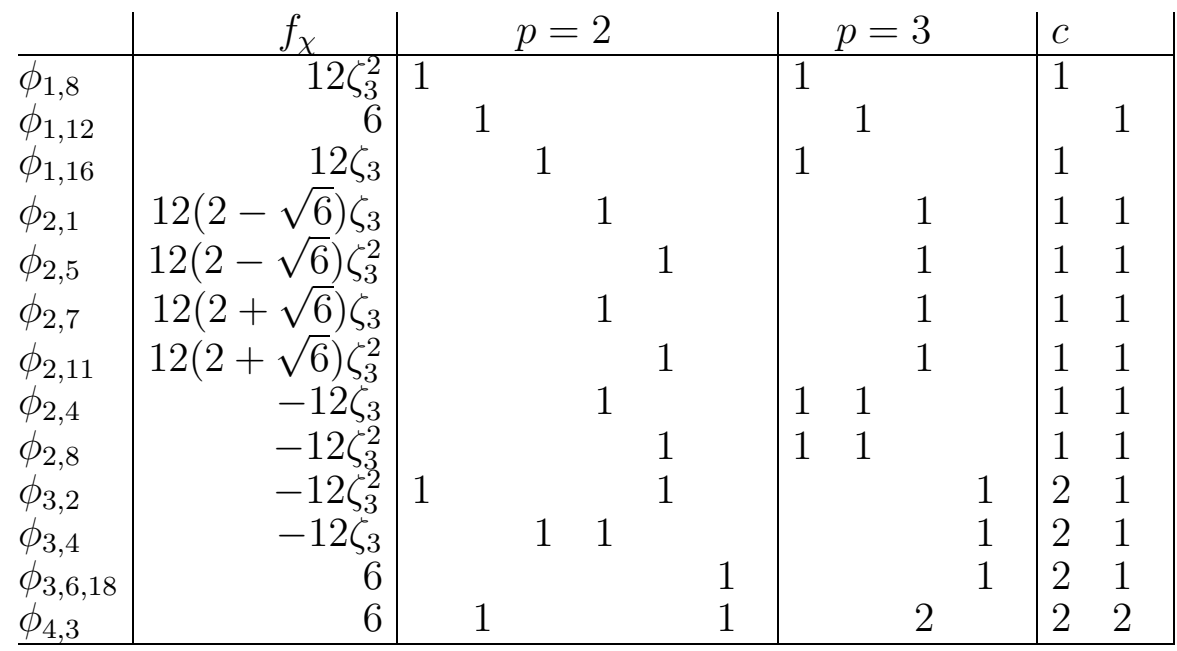

Avec les méthodes de 3.1, il restent deux questions pour la famille à 13 éléments. Pour $p=2$, on ne trouve pas le deuxième caractère projectif mais seulement la somme avec le sixième. Cependant, un calcul explicite montre que la représentation de caractère $\phi_{4,3}$ à des facteurs de degrés 3 et 1 modulo 2 et la matrice de décomposition est comme indiquée. Pour $p=3$, il faut montrer que le troisième projectif $\phi$ est indécomposable. On utilise alors le fait que les 4 caractères de degré 2 intervenant dans $\phi$ se factorisent par l'algèbre de Hecke $\mathcal{H}\left(G_{12}\right)$. Il est alors facile de voir qu'ils ont même réduction modulo 3.

3.7. $W=G_{23}=W\left(H_{3}\right)$. Le groupe de réflexions $G_{23}$ a 7 familles de caractères, des types suivants :

- défaut $0:\left(\phi_{1,0}\right),\left(\phi_{5,2}\right),\left(\phi_{5,5}\right),\left(\phi_{1,15}\right)$ 
- type $\alpha_{1^{2}}\left(2^{2}\right):\left(\phi_{4,3}, \phi_{4,4}\right)$

- type $\alpha_{1^{2}}(5):\left(\phi_{3,1}, \phi_{3,3}\right),\left(\phi_{3,6}, \phi_{3,8}\right)$

3.8. $W=G_{24}$. Le groupe de réflexions $G_{24}$ a 7 familles de caractères, des types suivants :

- défaut $0:\left(\phi_{1,0}\right),\left(\phi_{7,3}\right),\left(\phi_{7,6}\right),\left(\phi_{1,21}\right)$

- type $\alpha_{1^{2}}\left(2^{2}\right):\left(\phi_{8,4}, \phi_{8,5}\right)$

- type $\alpha_{G_{4}}(7):\left(\phi_{3,3}, \phi_{3,1}, \phi_{6,2}\right)\left(\phi_{3,8}, \phi_{3,10}, \phi_{6,9}\right)$

Pour établir ce resultat il faut voir que le projectif $\phi_{3,1}+\phi_{3,3}+2 \phi_{6,2}$ n'est pas indécomposable modulo 2 (et de même pour le produit avec le caractère déterminant). Puisque $\phi_{3,1}$ et $\phi_{3,3}$ ont des réductions différentes pour l'algèbre $k_{\mathfrak{p}}[W]$, alors il en est de même pour $\mathcal{H}$.

3.9. $W=G_{25}$. Pour distinguer les caractères de $W=G_{25}$ ayant mêmes valeurs $d, b$ nous choissisons la notation telle que $\left(\phi_{3,1}, \phi_{3,5}^{\prime}\right),\left(\phi_{3,5}^{\prime \prime}, \phi_{3,13}^{\prime \prime}\right),\left(\phi_{3,13}^{\prime}, \phi_{3,17}\right),\left(\phi_{6,2}, \phi_{6,4}^{\prime}\right)$, $\left(\phi_{6,4}^{\prime \prime}, \phi_{6,8}^{\prime \prime}\right)$ et $\left(\phi_{6,8}^{\prime}, \phi_{6,10}\right)$ sont des paires de caractères conjugués.

Le groupe de réflexions $G_{25}$ a 7 familles de caractères, des types suivants :

- défaut $0:\left(\phi_{1,0}\right)$

- type $\alpha_{1^{2}}(3):\left(\phi_{3,1}, \phi_{3,5}^{\prime}\right),\left(\phi_{8,6}, \phi_{8,9}\right),\left(\phi_{6,8}^{\prime}, \phi_{6,10}\right)$

- type $\mathcal{M}\left(Z_{3}\right)^{\prime}:\left(\phi_{2,3}, \phi_{2,9}, \phi_{6,2}, \phi_{6,4}^{\prime}, \phi_{8,3}\right)$

- type $\alpha_{3 \times 5}:\left(\phi_{3,5}^{\prime \prime}, \phi_{3,13}^{\prime \prime}, \phi_{3,6}, \phi_{6,4}^{\prime \prime}, \phi_{6,8}^{\prime \prime}, \phi_{9,5}, \phi_{9,7}\right)$

- Autre famille :

\begin{tabular}{|c|c|c|c|c|c|c|}
\hline & $f_{x}$ & & $p=2$ & \multicolumn{3}{|c|}{$p=3 \mid c$} \\
\hline$\phi_{1,12}$ & -6 & 1 & & & & 1 \\
\hline$\phi_{1,24}$ & -6 & & 1 & & & 1 \\
\hline$\phi_{2,15}$ & 6 & 1 & 1 & & & 2 \\
\hline$\phi_{3,13}^{\prime}$ & $-3 \zeta_{3}$ & & 1 & & & 3 \\
\hline$\phi_{3,17}$ & $-3 \zeta_{3}^{2}$ & & & & 1 & 3 \\
\hline
\end{tabular}

Plusieurs points sont à justifier. Tout d'abord, il faut démontrer qu'il y a un module simple de dimension 6 dans la famille $\mathcal{M}\left(Z_{3}\right)^{\prime}$ pour $p=3$.

Supposons $p=3$. Soit $C$ le bloc de $\mathcal{O}_{\mathfrak{p}} \mathcal{H}$ correspondant à la famille de type $\mathcal{M}\left(Z_{3}\right)^{\prime}$. Soit $W^{\prime}$ un sous-groupe parabolique de $W$ de type $G(3,1,1) \times G(3,1,1)$, de générateurs $s, u$ (conjugués dans $W$ ) et $\mathcal{H}^{\prime}$ son algèbre de Hecke.

Le lemme 2.7 montre que l'algèbre $C$ est projective relativement à $\mathcal{O}_{\mathfrak{p}} \mathcal{H}^{\prime}$.

Soit $S$ un $\mathcal{O}_{\mathfrak{p}} \mathcal{H}$-module libre sur $\mathcal{O}_{\mathfrak{p}}$ tel que $K \otimes_{\mathcal{O}_{\mathfrak{p}}} S$ a pour caractère $\phi_{2,3}$. Soit $\bar{S}$ sa réduction modulo 3 . C'est un $k_{\mathfrak{p}} \mathcal{H}$-module simple.

Soit $T$ le $C$-module simple non isomorphe à $\bar{S}$. Nous allons montrer que $T$ est de dimension 6 .

On note $\psi_{i, j}$ le caractère $s \mapsto \operatorname{det}(s)^{i}, u \mapsto \operatorname{det}(t)^{j}$ de $W^{\prime}$.

Soit $Y=C \otimes_{\mathcal{O}_{\mathfrak{p}} \mathcal{H}^{\prime}} L$, où $L$ est le $\mathcal{O}_{\mathfrak{p}} \mathcal{H}^{\prime}$-module libre de rang 1 sur $\mathcal{O}_{\mathfrak{p}}$ tel que $K \otimes_{\mathcal{O}_{\mathfrak{p}}} L$ est de caractère $\psi_{1,1}$. Alors, le caractère de $Y$ est $\phi_{2,3}+\phi_{6,2}$. On a $\operatorname{Res}_{\mathcal{H}^{\prime}} S \stackrel{\sim}{\longrightarrow} L \oplus P$, où $P$ est le $\mathcal{O}_{\mathfrak{p}} \mathcal{H}^{\prime}$-module projectif de caractère $\phi_{0,0}$.

Puisque $C$ est projective relativement à $\mathcal{O}_{\mathfrak{p}} \mathcal{H}^{\prime}$, la surjection canonique

$$
C \otimes_{\mathcal{O}_{\mathfrak{p}} \mathcal{H}^{\prime}} \operatorname{Res}_{\mathcal{H}^{\prime}} S \rightarrow S
$$

est scindée. On en déduit que $S \mid Y \oplus$ projectif, donc que $S \mid Y$. Soit $Y^{\prime}$ tel que $Y \simeq$ $S \oplus Y^{\prime}$. On a $\operatorname{Hom}(\bar{Y}, \bar{S}) \simeq \operatorname{Hom}\left(\bar{L}, \operatorname{Res}_{\mathcal{H}^{\prime}} \bar{S}\right) \simeq k_{\mathfrak{p}}$. On en déduit que $\operatorname{Hom}\left(\bar{Y}^{\prime}, \bar{S}\right)=0$. 
De même, $\operatorname{Hom}\left(\bar{S}, \bar{Y}^{\prime}\right)=0$. Puisque la multiplicité de $T$ dans $\bar{Y}^{\prime}$ est 1 , on en déduit que $\bar{Y}^{\prime}=T$. Ceci montre que $T$ est de dimension 6 .

Pour achever la démonstration des résultats annoncés plus haut, il reste à prouver que $\phi_{9,5}$ et $\phi_{9,7}$ ne sont pas dans le même 3 -bloc que $\phi_{3,5}^{\prime \prime}, \phi_{3,13}^{\prime \prime}, \phi_{3,6}, \phi_{6,4}^{\prime \prime}$ et $\phi_{6,8}^{\prime \prime}$. Nous le déduisons (cf. lemme 2.7) du fait que $\operatorname{Tr}_{\mathcal{H}^{\prime}}^{\mathcal{H}}(1)$ agit par un scalaire inversible de $\mathcal{O}_{\mathfrak{p}}$ sur les 5 derniers caractères, mais pas sur $\phi_{9,5}$ et $\phi_{9,7}$.

3.10. $W=G_{26}$. Pour distinguer les caractères de $W=G_{26}$ ayant mêmes $d, b$, nous choissisons la notation telle que $\left(\phi_{3,1}, \phi_{3,5}^{\prime}\right),\left(\phi_{3,5}^{\prime \prime}, \phi_{3,13}^{\prime \prime}\right),\left(\phi_{3,13}^{\prime}, \phi_{3,17}\right),\left(\phi_{3,4}, \phi_{3,8}^{\prime}\right)$, $\left(\phi_{3,8}^{\prime \prime}, \phi_{3,16}^{\prime \prime}\right),\left(\phi_{3,16}^{\prime}, \phi_{3,20}\right),\left(\phi_{6,2}, \phi_{6,4}^{\prime}\right),\left(\phi_{6,4}^{\prime \prime}, \phi_{6,8}^{\prime \prime}\right),\left(\phi_{6,8}^{\prime}, \phi_{6,10}\right),\left(\phi_{6,5}, \phi_{6,7}^{\prime}\right),\left(\phi_{6,7}^{\prime \prime}, \phi_{6,11}^{\prime \prime}\right)$ et $\left(\phi_{6,11}^{\prime}, \phi_{6,13}\right)$ sont des paires de caractères conjugués. De plus, $R_{\chi} \equiv x^{24}\left(\bmod x^{23}\right)$ pour $\chi=\phi_{8,6}^{\prime}$ et $R_{\chi} \equiv x^{27}\left(\bmod x^{26}\right)$ pour $\chi=\phi_{8,9}^{\prime}$.

Le groupe de réflexions $G_{26}$ a 10 familles de caractères, des types suivants :

- défaut $0:\left(\phi_{1,0}\right)$

- type $\alpha_{1^{2}}\left(2^{2}\right):\left(\phi_{8,3}, \phi_{8,6}^{\prime}\right)$

- type $\alpha_{1^{2}}(3):\left(\phi_{6,5}, \phi_{6,7}^{\prime}\right),\left(\phi_{3,16}^{\prime}, \phi_{3,20}\right)$

- type $\alpha_{G_{4}}(3):\left(\phi_{1,21}, \phi_{1,33}, \phi_{2,24}\right)$

- type $\mathcal{M}\left(Z_{3}\right):\left(\phi_{2,3}, \phi_{2,9}, \phi_{1,9}, \phi_{3,1}, \phi_{3,5}^{\prime}\right),\left(\phi_{3,4}, \phi_{3,8}^{\prime}, \phi_{3,6}, \phi_{6,4}^{\prime}, \phi_{6,2}\right),\left(\phi_{3,13}^{\prime}, \phi_{3,17}, \phi_{3,15}\right.$, $\left.\phi_{6,13}, \phi_{6,11}^{\prime}\right)$

- Autres familles :

\begin{tabular}{|c|c|c|c|c|}
\hline & $f_{\chi}$ & $p=2$ & $p=3$ & l $c$ \\
\hline$\overline{\phi_{3,5}^{\prime \prime}}$ & $-3-\sqrt{-3}$ & 1 & 1 & 1 \\
\hline$\phi_{3,13}^{\prime \prime}$ & $-3+\sqrt{-3}$ & 1 & 1 & 1 \\
\hline$\phi_{6,4}^{\prime \prime}$ & $3-\sqrt{-3}$ & 1 & 1 & 11 \\
\hline$\phi_{6,8}^{\prime \prime}$ & $3+\sqrt{-3}$ & 1 & 1 & 11 \\
\hline$\phi_{9,7}$ & $3-\sqrt{-3}$ & 11 & 1 & 21 \\
\hline$\phi_{9,5}$ & $3+\sqrt{-3}$ & 1 & 1 & 21 \\
\hline
\end{tabular}




\begin{tabular}{|c|c|c|c|c|c|c|c|c|c|}
\hline & $f_{\chi}$ & & $p=$ & & & & $=3$ & & $c$ \\
\hline 1,12 & $18 \sqrt{-3}$ & 1 & & & & 1 & & & 1 \\
\hline & $-18 \sqrt{-3}$ & & 1 & & & 1 & & & 1 \\
\hline & & & & & & & & & 2 \\
\hline 18 & $9 \sqrt{-3}$ & & & & & & 1 & & 1 \\
\hline 2,12 & $-9 \sqrt{-3}$ & & & & & & 1 & & 1 \\
\hline 3,8 & $3(3-\sqrt{-3})$ & & 1 & & & & 1 & & 11 \\
\hline "I,16 & $3(3+\sqrt{-3})$ & & 1 & & & & 1 & & 11 \\
\hline 6,8 & $3 \sqrt{-3} \zeta_{3}$ & & & & & & & & \\
\hline 6,10 & $-3 \sqrt{-3} \zeta_{3}^{2}$ & & & & & & 1 & & 3 \\
\hline 6,11 & $-6 \zeta_{3}^{2}$ & & & 1 & & & & 1 & 11 \\
\hline 6,7 & $-6 \zeta_{3}$ & & & 1 & & & & 1 & 11 \\
\hline 8,9 & 6 & & & 1 & & & & 1 & 31 \\
\hline 8,9 & 6 & & & & & & & 1 & 31 \\
\hline 8,12 & $18 \sqrt{-3}$ & & & & & 1 & 11 & & 31 \\
\hline 8,6 & $-18 \sqrt{-3}$ & & & 1 & & 1 & 11 & & 31 \\
\hline 9,8 & $3(3-\sqrt{-3})$ & & 1 & & & & 21 & & 22 \\
\hline & $3(3+\sqrt{-3})$ & & 1 & 1 & & & 21 & & 22 \\
\hline
\end{tabular}

Dans la table nous avons omis les projectifs appartenant à des blocs de $p$-défaut 0 .

3.11. $W=G_{27}$. Le groupe de réflexions complexe $G_{27}$ a cinq paires de caractères de mêmes degré et invariant $b$. Nous distinguons ceux de degré 3 et 8 en donnant comme troisième indice le degré en $x$ du degré fantôme.

Le produit par le caractère déterminant $\epsilon$ donne un automorphisme des matrices de décomposition. Nous utilisons ce fait en donnant le résultat pour $\chi$ et $\chi \otimes \epsilon$ dans la même ligne.

Le groupe de réflexions $G_{27}$ a 11 familles de caractères, des types suivants :

- défaut $0:\left(\phi_{1,0}\right),\left(\phi_{1,45}\right)$

- type $\alpha_{1^{2}}(3):\left(\phi_{15,7}, \phi_{15,5}\right),\left(\phi_{15,8}, \phi_{15,10}\right)$

- type $\mathcal{M}\left(Z_{2}\right):\left(\phi_{5,6}^{\prime}, \phi_{5,6}^{\prime \prime}, \phi_{10,3}\right),\left(\phi_{5,15}^{\prime \prime}, \phi_{5,15}^{\prime}, \phi_{10,12}\right)$

- type $\alpha_{1^{3}}(3):\left(\phi_{9,4}, \phi_{9,8}, \phi_{9,6}\right),\left(\phi_{9,9}, \phi_{9,11}, \phi_{9,13}\right)$

- type $\alpha_{3 \times 4}(5)$ : $\left(\phi_{8,6}, \phi_{8,9,39}, \phi_{8,9,33}, \phi_{8,12}\right)$

- Autre famille :

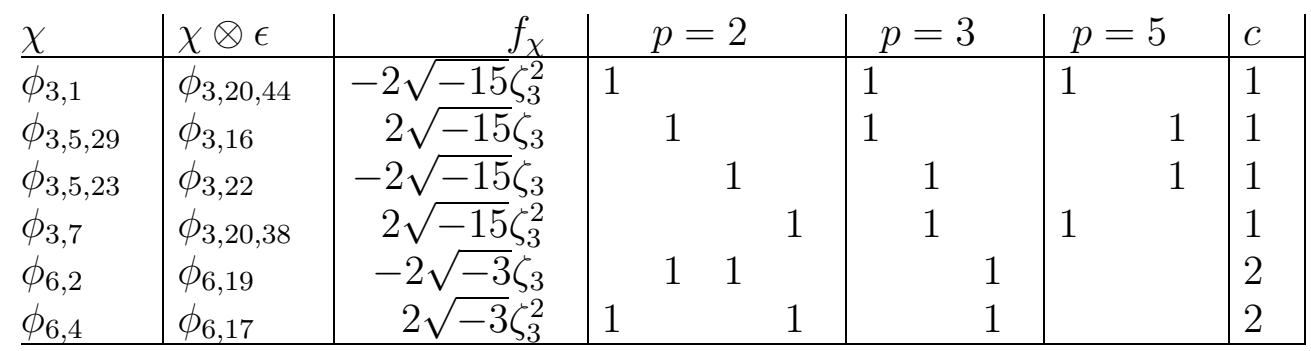

3.12. $W=G_{28}=W\left(F_{4}\right)$. Les résultats ont été obtenus par Gyoja [13]. Pour les caractères de $W\left(F_{4}\right)$, nous utilisons la notation standard (cf [11, Table C.3]).

Il y a 11 familles de caractères, des types suivants :

- type $\mathcal{M}\left(Z_{2}\right):\left(\phi_{4,1}, \phi_{2,4}^{\prime \prime}, \phi_{2,4}^{\prime}\right),\left(\phi_{4,13}, \phi_{2,16}^{\prime}, \phi_{2,16}^{\prime \prime}\right)$ 
- Autre famille :

\begin{tabular}{|c|c|c|c|c|c|c|c|c|c|c|c|c|c|}
\hline & $f_{\chi}$ & \multicolumn{5}{|c|}{$p=2$} & \multicolumn{2}{|c|}{$p=3$} & \multicolumn{5}{|c|}{$c$} \\
\hline $\bar{\phi}_{1,12}^{\prime \prime}$ & 8 & 1 & & & & & & & 1 & & & & \\
\hline$\phi_{1,12}^{\prime}$ & 8 & & 1 & & & & & & & 1 & & & \\
\hline$\phi_{4,7}^{\prime \prime \prime}$ & 4 & & & & & & & & 1 & & 1 & & \\
\hline$\phi_{4,7}^{\prime}$ & 4 & & & & 1 & & & & & 1 & & 1 & \\
\hline$\phi_{4,8}$ & 8 & & & & & 1 & & & & & & & 1 \\
\hline$\phi_{6,6}^{\prime}$ & 3 & & & & & & 1 & & & & 1 & 1 & \\
\hline$\phi_{6,6}^{\prime \prime}$ & 12 & 1 & 1 & & & 1 & & 1 & 1 & 1 & & & 1 \\
\hline$\phi_{9,6}^{\prime \prime \prime}$ & 8 & 1 & & 1 & & 1 & & & 2 & & 1 & & 1 \\
\hline$\phi_{9,6}^{\prime}$ & 8 & & 1 & & 1 & 1 & & & & 2 & & 1 & 1 \\
\hline$\phi_{124}$ & 24 & & & 1 & 1 & 1 & 1 & 1 & 1 & 1 & 1 & 1 & 1 \\
\hline$\phi_{16,5}$ & 4 & & & 1 & 1 & 2 & & & 1 & 1 & 1 & 1 & 2 \\
\hline
\end{tabular}

Tous les autres familles sont de défaut 0 .

3.13. $W=G_{29}$. Pour $W=G_{29}$, nous notons $\phi_{6,10}^{\prime}, \phi_{6,10}^{\prime \prime}$ les deux caractères réels de degré 6 et d'invariant $b$ égal à 10 . En outre, $\phi_{15,4}^{\prime \prime}$ est le caractère de degré 15 intervenant dans $\phi_{4,3} \otimes \phi_{4,1}$ et $\phi_{15,12}^{\prime \prime}=\phi_{15,4}^{\prime \prime} \otimes \epsilon$ avec $\epsilon=\phi_{1,40}$ le caractère déterminant.

Le groupe de réflexions $G_{29}$ a 15 familles de caractères, des types suivants :

- défaut $0:\left(\phi_{1,0}\right),\left(\phi_{10,2}\right),\left(\phi_{15,4}^{\prime}\right),\left(\phi_{15,12}^{\prime}\right),\left(\phi_{10,18}\right),\left(\phi_{1,40}\right)$

- type $\alpha_{1^{3}}(2):\left(\phi_{4,3}, \phi_{4,4}, \phi_{4,1}\right),\left(\phi_{20,5}, \phi_{20,6}, \phi_{20,7}\right),\left(\phi_{20,11}, \phi_{20,10}, \phi_{20,9}\right),\left(\phi_{4,21}, \phi_{4,24}, \phi_{4,23}\right)$

- type $\alpha_{1^{2}}\left(2^{2}\right)$ : $\left(\phi_{16,3}, \phi_{16,5}\right),\left(\phi_{16,13}, \phi_{16,15}\right)$

- type $\mathcal{M}\left(Z_{2}\right):\left(\phi_{5,8}, \phi_{10,6}, \phi_{15,4}^{\prime \prime}\right),\left(\phi_{5,16}, \phi_{10,14}, \phi_{15,12}^{\prime \prime}\right)$

- Autre famille :

\begin{tabular}{|c|c|c|c|c|c|c|c|c|c|c|}
\hline$\underline{\chi}$ & $f_{\chi}$ & \multicolumn{2}{|l|}{$p=2$} & \multicolumn{4}{|c|}{$p=5$} & \multicolumn{3}{|c|}{$c$} \\
\hline $\bar{\phi}_{6,10}^{\prime}$ & 5 & 1 & & 1 & & & & 1 & & \\
\hline$\phi_{6,10}^{\prime \prime}$ & 5 & 1 & & & 1 & & & & 1 & \\
\hline$\phi_{6,10}^{\prime \prime \prime}$ & -20 & 1 & & & & 1 & & & & 1 \\
\hline$\phi_{6,10}^{\prime \prime \prime \prime}$ & -20 & 1 & & & & & 1 & & & 1 \\
\hline 6,12 & 4 & 1 & & & & & & & & 1 \\
\hline$\phi_{24,6}$ & 20 & 2 & 1 & 1 & 1 & 1 & 1 & 1 & 1 & 2 \\
\hline$\phi_{24,7}$ & 4 & 2 & 1 & & & & & 1 & 1 & 2 \\
\hline$\phi_{24,9}$ & 4 & 2 & 1 & & & & & 1 & 1 & 2 \\
\hline$\phi_{30,8}$ & 4 & 3 & 1 & & & & & 1 & 1 & 3 \\
\hline & & $*$ & & & & & & & & \\
\hline
\end{tabular}

Pour $W=G_{29}$ nous ne savons pas démontrer que le troisième caractère projectif modulo 2 dans la famille à 9 éléments est indécomposable.

Nous l'indiquons par l'* sous la colonne correspondante de la table.

3.14. $W=G_{30}=W\left(H_{4}\right)$. Pour $W=G_{30}$ le caractère $\phi_{30,10}^{\prime}$ de degré 30 est celui pour lequel $\phi_{30,10}^{\prime}(c)=\phi_{4,1}(c)$ pour un élément de Coxeter $c \in W$, tandis que $\phi_{30,10}^{\prime \prime}(c)=$ $\phi_{4,7}(c)$.

Le groupe de réflexions $G_{30}$ a 13 familles de caractères, des types suivants :

- défaut 0 : $\left(\phi_{1,0}\right),\left(\phi_{25,4}\right),\left(\phi_{36,5}\right),\left(\phi_{36,15}\right),\left(\phi_{25,16}\right),\left(\phi_{1,60}\right)$

- type $\alpha_{1^{2}}(5):\left(\phi_{4,1}, \phi_{4,7}\right),\left(\phi_{9,2}, \phi_{9,6}\right),\left(\phi_{9,22}, \phi_{9,26}\right),\left(\phi_{4,31}, \phi_{4,37}\right)$

- type $\alpha_{1^{2}}\left(2^{2}\right):\left(\phi_{16,3}, \phi_{16,6}\right),\left(\phi_{16,18}, \phi_{16,21}\right)$ 
- Autre famille :

\begin{tabular}{|c|c|c|c|c|c|c|c|c|c|c|}
\hline$\chi$ & $f_{\chi}$ & \multicolumn{2}{|r|}{$p=2$} & $p=3$ & \multicolumn{3}{|c|}{$p=5$} & \multicolumn{3}{|c|}{$c$} \\
\hline$\phi_{6,12}$ & $15(3+\sqrt{5})$ & 1 & & 1 & 1 & & & 1 & & \\
\hline 6,20 & $15(3-\sqrt{5})$ & 1 & & 1 & 1 & & & 1 & & \\
\hline 3,13 & 10 & & & & & 1 & & & 1 & \\
\hline 0,12 & 10 & & 1 & & & 1 & & & & 1 \\
\hline$b_{18,10}$ & 10 & & 1 & & & 1 & & & 1 & 1 \\
\hline$\phi_{24,7}$ & $15(7+3 \sqrt{5})$ & & 1 & 1 & & & 1 & 1 & 1 & 1 \\
\hline$\phi_{24,11}$ & $15(7-3 \sqrt{5})$ & & 1 & 1 & & 1 & 1 & 1 & 1 & 1 \\
\hline$\phi_{30,10}^{\prime}$ & $15(3-\sqrt{5})$ & 1 & 1 & 1 & & & 1 & 2 & 1 & 1 \\
\hline$\phi_{30,10}^{\prime \prime}$ & $15(3+\sqrt{5})$ & 1 & 1 & 1 & & & 1 & 2 & 1 & 1 \\
\hline$\phi_{8,12}$ & 8 & & 1 & & & & & & 1 & \\
\hline$\phi_{16,11}$ & 20 & & 1 & & & & 1 & 1 & & 1 \\
\hline$\phi_{16,13}$ & 20 & & 1 & & & & 1 & 1 & & 1 \\
\hline$\phi_{24,6}$ & $120(9+4 \sqrt{5})$ & & 11 & 1 & 1 & 1 & & 1 & 1 & 1 \\
\hline$\phi_{24,12}$ & $120(9-4 \sqrt{5})$ & & 11 & 1 & 1 & 1 & & 1 & 1 & 1 \\
\hline$b_{10}$ & & & 111 & & & 1 & 1 & 2 & 1 & 2 \\
\hline$\phi_{48,9}$ & 12 & & 211 & 11 & & & & & 2 & 2 \\
\hline
\end{tabular}

En utilisant le logiciel MeatAxe, Jürgen Müller a démontré que les caractères de dégre 30 restent irréductibles modulo 5 .

Les caractères constructibles de $W\left(H_{4}\right)$ sont déjà dans Alvis-Lusztig [1].

3.15. $W=G_{32}$. Pour distinguer les caractères de $W=G_{32}$ ayant même valeurs $d, b$, nous choisissons la notation telle que $\left(\phi_{20,3}, \phi_{20,9}^{\prime}\right),\left(\phi_{20,9}^{\prime \prime}, \phi_{20,21}\right),\left(\phi_{20,13}, \phi_{20,29}^{\prime}\right)$, $\left(\phi_{20,29}^{\prime \prime}, \phi_{20,31}\right),\left(\phi_{30,12}^{\prime}, \phi_{30,24}\right),\left(\phi_{30,16}, \phi_{30,20}^{\prime}\right),\left(\phi_{30,20}^{\prime \prime}, \phi_{30,28}\right),\left(\phi_{60,9}, \phi_{60,15}^{\prime}\right),\left(\phi_{60,7}, \phi_{60,11}^{\prime}\right)$ et $\left(\phi_{60,11}^{\prime \prime}, \phi_{60,13}\right)$ sont des paires de caractères conjugués.

Le groupe de réflexions $G_{32}$ a 16 familles de caractères, des types suivants :

- défaut $0:\left(\phi_{1,0}\right)$

- type $\alpha_{1^{2}}(3):\left(\phi_{4,1}, \phi_{4,11}\right),\left(\phi_{20,3}, \phi_{20,9}^{\prime}\right)$,

- type $\alpha_{G_{4}}(3):\left(\phi_{10,30}, \phi_{10,42}, \phi_{20,33}\right)$

- type $\alpha_{3 \times 5}:\left(\phi_{6,8}, \phi_{6,28}, \phi_{24,6}, \phi_{30,4}, \phi_{30,8}, \phi_{36,5}, \phi_{36,7}\right),\left(\phi_{15,22}, \phi_{15,38}, \phi_{15,24}, \phi_{30,20}^{\prime \prime}\right.$, $\left.\phi_{30,28}, \phi_{45,22}, \phi_{45,26}\right)$

- type $\alpha_{3 \times 5}^{\prime}:\left(\phi_{20,5}, \phi_{20,19}, \phi_{20,7}, \phi_{20,17}, \phi_{40,8}, \phi_{40,10}\right)$,

- type $\alpha_{3 \times 4}(3):\left(\phi_{64,13}, \phi_{64,16}, \phi_{64,8}, \phi_{64,11}\right)$

- type $\mathcal{M}\left(Z_{3}\right)^{\prime}:\left(\phi_{5,20}, \phi_{5,4}, \phi_{10,2}, \phi_{10,10}, \phi_{15,6}\right)$

- type $\alpha_{1^{4}}(3):\left(\phi_{20,25}, \phi_{20,29}^{\prime \prime}, \phi_{20,31}, \phi_{20,35}\right),\left(\phi_{60,9}, \phi_{60,11}^{\prime \prime}, \phi_{60,13}, \phi_{60,15}^{\prime}\right)$

- type $\alpha_{1^{3}}(3):\left(\phi_{81,10}, \phi_{81,12}, \phi_{81,14}\right)$

- Autres familles : 


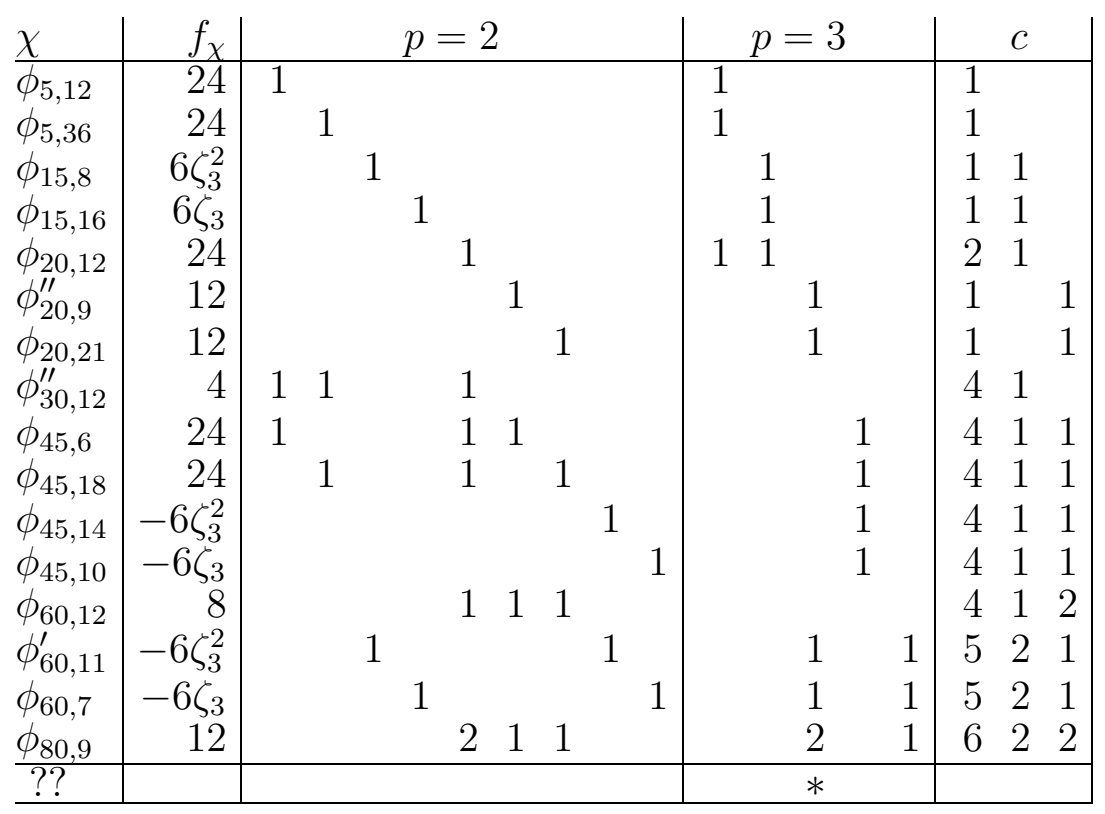

\begin{tabular}{|c|c|c|c|c|c|c|c|}
\hline x & $f_{\chi}$ & $p=2$ & & & $=3$ & & \\
\hline$\phi_{10,34}$ & $-6 \sqrt{-3}$ & 1 & . & 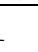 & & & \\
\hline$\phi_{20,13}$ & -6 & 1 & & 1 & & & \\
\hline$\phi_{20,16}$ & $6 \sqrt{-3}$ & 1 & & & 1 & & . \\
\hline$\phi_{30,16}$ & $-6 \sqrt{-3}$ & 11 & & & 1 & 2 & \\
\hline$\phi_{60,16}$ & $-6 \sqrt{-3}$ & 1 & & & 11 & & \\
\hline$b_{80,13}$ & & 11 & & & & 3 & \\
\hline$\phi_{10,14}$ & $6 \sqrt{-3}$ & 1 & & 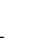 & & L & \\
\hline$\phi_{20,29}^{\prime}$ & -6 & 1 & & 1 & & & \\
\hline$\phi_{20,20}$ & $-6 \sqrt{-3}$ & 1 & & & 1 & & \\
\hline$\phi_{30,20}^{\prime}$ & $6 \sqrt{-3}$ & 11 & & & 1 & 2 & \\
\hline$\phi_{60,20}$ & $6 \sqrt{-3}$ & 1 & & & 11 & 3 & \\
\hline$\phi_{80,17}$ & & 11 & & & & 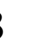 & \\
\hline$\phi_{30,12}^{\prime}$ & $-6 \sqrt{-3}$ & 1 & & L & 1 & 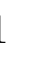 & \\
\hline$\phi_{30,24}$ & $6 \sqrt{-3}$ & & & 1 & 1 & L & \\
\hline$\phi_{60,15}^{\prime \prime}$ & & & & & & 2 & 2 \\
\hline$\phi_{40,14}$ & $-3 \sqrt{-3}$ & & & & 1 & & \\
\hline$\phi_{40,22}$ & $3 \sqrt{-3}$ & & & & 1 & & \\
\hline & & & & k & $* *$ & & \\
\hline
\end{tabular}

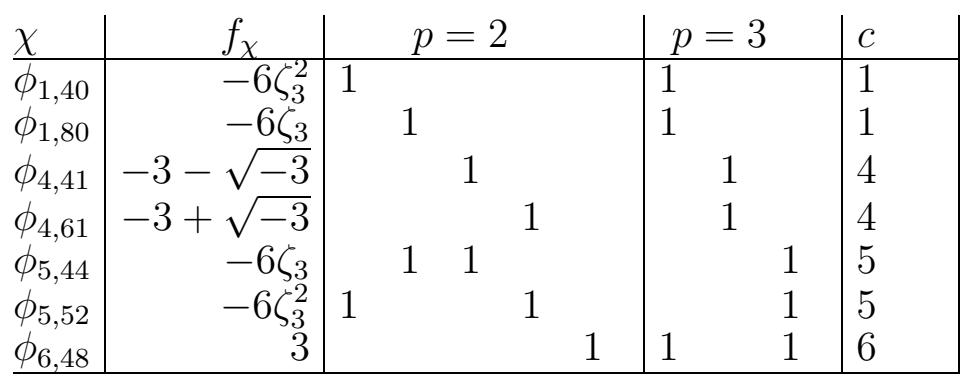




\begin{tabular}{|c|c|c|c|c|c|c|c|c|c|c|c|c|}
\hline$\chi$ & $f_{\chi}$ & \multicolumn{4}{|c|}{$p=2$} & \multicolumn{2}{|c|}{$p=3$} & \multicolumn{3}{|c|}{$p=5$} & \multicolumn{2}{|c|}{$c$} \\
\hline $\bar{\phi}_{4,21}$ & 30 & 1 & & & & 1 & & & & & 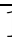 & \\
\hline$\phi_{4,51}$ & 30 & & 1 & & & 1 & & & 1 & & & \\
\hline$\phi_{24,16}$ & $6 \zeta_{3}^{2}$ & & 1 & & & & 1 & & & & & \\
\hline$\phi_{24,26}$ & $6 \zeta_{3}$ & & & 1 & & & 1 & & & & & \\
\hline$\phi_{36,15}$ & -30 & 1 & & 1 & & & & 1 & 1 & & & \\
\hline$\phi_{36,17}$ & $-6 \zeta_{3}$ & & & 1 & & & & & & & & \\
\hline$\phi_{36,25}$ & $-6 \zeta_{3}^{2}$ & & & & 1 & 2 & 1 & & & & & \\
\hline$\phi_{36,27}$ & -30 & & & & 1 & 2 & 1 & & 1 & 1 & & \\
\hline$\phi_{40,24}$ & 6 & 1 & & & 1 & & & & & & & \\
\hline$\phi_{40,18}$ & & 1 & & 1 & & & & & & & & \\
\hline$\phi_{60,17}$ & $-6 \zeta_{3}$ & & & 1 & & & 2 & & & & & \\
\hline$\phi_{60,19}$ & $-6 \zeta_{3}^{2}$ & & 1 & & 1 & & 2 & & & & & \\
\hline$\phi_{64,18}$ & 6 & & & & & & 1 & & & & & , \\
\hline$\phi_{64,21}$ & 30 & & & & & 2 & 2 & & & 1 & & \\
\hline$? ?$ & & $*$ & & & & $*$ & $*$ & & & & & \\
\hline
\end{tabular}

Nous avons utilisé ici la matrice de décomposition (pour $p=3$ ) du groupe de réflexion $G_{32} \cong 3 \times 2 \cdot U_{4}(2)$ pour obtenir l'information manquante sur les quatre dernières familles, via le lemme 2.6(c).

3.16. $W=G_{33}$. Pour $W=G_{33}$, on note $\chi=\phi_{10,8}^{\prime}$ tel que $R_{\chi} \equiv x^{28}+x^{26}\left(\bmod x^{25}\right)$, et $\chi=\phi_{40,5}^{\prime}$ tel que $R_{\chi} \equiv x^{31}+x^{29}\left(\bmod x^{28}\right)$, et on note $\phi_{10,17}^{\prime}=\phi_{10,8} \otimes \epsilon, \phi_{40,14}^{\prime}=$ $\phi_{40,5} \otimes \epsilon$, avec $\epsilon=\phi_{1,45}$.

Le groupe de réflexions $G_{33}$ a 19 familles de caractères, des types suivants :

- défaut 0 : $\left(\phi_{1,0}\right),\left(\phi_{15,2}\right),\left(\phi_{81,6}\right),\left(\phi_{60,7}\right),\left(\phi_{15,9}\right),\left(\phi_{60,10}\right),\left(\phi_{81,11}\right),\left(\phi_{15,12}\right),\left(\phi_{15,23}\right)$, $\left(\phi_{1,45}\right)$

- type $\alpha_{1^{2}}(3):\left(\phi_{5,3}, \phi_{5,1}\right),\left(\phi_{45,9}, \phi_{45,7}\right),\left(\phi_{45,10}, \phi_{45,12}\right),\left(\phi_{5,28}, \phi_{5,30}\right)$

- type $\alpha_{1^{2}}\left(2^{2}\right):\left(\phi_{64,8}, \phi_{64,9}\right)$

- type $\mathcal{M}\left(Z_{2}\right):\left(\phi_{6,5}, \phi_{24,4}, \phi_{30,3}\right),\left(\phi_{6,20}, \phi_{24,19}, \phi_{30,18}\right)$

- type $\alpha_{3 \times 5}:\left(\phi_{10,8}^{\prime}, \phi_{10,8}^{\prime \prime}, \phi_{20,6}, \phi_{30,6}, \phi_{30,4}, \phi_{40,5}^{\prime}, \phi_{40,5}^{\prime \prime}\right),\left(\phi_{10,17}^{\prime \prime}, \phi_{10,17}^{\prime}, \phi_{20,15}, \phi_{30,13}\right.$, $\left.\phi_{30,15}, \phi_{40,14}^{\prime \prime}, \phi_{40,14}^{\prime}\right)$

3.17. $W=G_{34}$. Les notations utilisées pour les caractères irréductibles de $G_{34}$ sont celles de [21, 4C].

Le groupe de réflexions $G_{34}$ a 40 familles de caractères, des types suivants :

- défaut $0:\left(\phi_{1,0}\right),\left(\phi_{70,9}^{\prime}\right),\left(\phi_{210,12}\right),\left(\phi_{210,30}\right),\left(\phi_{70,45}^{\prime}\right),\left(\phi_{1,126}\right)$

- type $\alpha_{1^{2}}(3):\left(\phi_{6,5}, \phi_{6,1}\right),\left(\phi_{21,4}, \phi_{21,2}\right),\left(\phi_{630,13}, \phi_{630,11}\right),\left(\phi_{630,14}, \phi_{630,16}\right),\left(\phi_{630,22}\right.$, $\left.\phi_{630,20}\right),\left(\phi_{630,23}, \phi_{630,25}\right),\left(\phi_{21,68}, \phi_{21,70}\right),\left(\phi_{6,85}, \phi_{6,89}\right)$

- type $\mathcal{M}\left(Z_{2}\right):\left(\phi_{21,6}, \phi_{35,6}, \phi_{56,3}\right),\left(\phi_{140,12}, \phi_{420,12}, \phi_{560,9}\right),\left(\phi_{140,21}, \phi_{420,21}, \phi_{560,18}^{\prime}\right)$, $\left(\phi_{140,30}, \phi_{420,30}, \phi_{560,27}\right),\left(\phi_{21,60}, \phi_{35,60}, \phi_{56,57}\right)$

- type $\alpha_{1^{2}}\left(2^{2}\right):\left(\phi_{896,12}, \phi_{896,15}\right),\left(\phi_{896,21}, \phi_{896,24}\right)$

- type $\alpha_{3 \times 5}:\left(\phi_{15,16}, \phi_{15,14}, \phi_{90,6}, \phi_{105,8}^{\prime}, \phi_{105,4}, \phi_{120,7}, \phi_{120,5}\right),\left(\phi_{15,56}, \phi_{15,58}, \phi_{90,48}\right.$, $\left.\phi_{105,46}, \phi_{105,50}, \phi_{120,47}, \phi_{120,49}\right)$

- type $\mathcal{M}\left(Z_{3}\right):\left(\phi_{70,9}^{\prime \prime \prime}, \phi_{70,9}^{\prime \prime}, \phi_{56,9}, \phi_{126,5}, \phi_{126,7}\right),\left(\phi_{280,12}^{\prime \prime}, \phi_{280,12}^{\prime}, \phi_{35,18}, \phi_{315,10}, \phi_{315,14}\right)$, $\left(\phi_{210,13}, \phi_{210,17}, \phi_{630,15}, \phi_{840,11}, \phi_{840,13}^{\prime}\right),\left(\phi_{210,29}, \phi_{210,25}, \phi_{630,27}, \phi_{840,23}^{\prime \prime}, \phi_{840,25}\right)$, $\left(\phi_{280,30}^{\prime}, \phi_{280,30}^{\prime \prime}, \phi_{35,36}, \phi_{315,28}, \phi_{315,32}\right),\left(\phi_{70,45}^{\prime \prime}, \phi_{70,45}^{\prime \prime \prime}, \phi_{56,45}, \phi_{126,41}, \phi_{126,43}\right)$

- type $\mathcal{M}\left(Z_{3}\right)^{\prime \prime}:\left(\phi_{105,8}^{\prime}, \phi_{105,10}, \phi_{210,8}, \phi_{210,10}, \phi_{315,6}\right),\left(\phi_{105,38}, \phi_{105,40}, \phi_{210,38}, \phi_{210,40}\right.$, $\left.\phi_{315,36}\right)$ 
- type $\alpha_{3 \times 5}^{\prime \prime}:\left(\phi_{84,13}, \phi_{84,17}, \phi_{336,8}, \phi_{336,10}, \phi_{420,11}, \phi_{420,7}, \phi_{504,9}\right),\left(\phi_{84,41}, \phi_{84,37}, \phi_{336,34}\right.$, $\left.\phi_{336,32}, \phi_{420,35}, \phi_{420,33}, \phi_{504,31}\right)$,

- type $\alpha_{3 \times 4}(3):\left(\phi_{384,8}, \phi_{384,11}, \phi_{384,13}, \phi_{384,10}\right),\left(\phi_{384,34}, \phi_{384,31}, \phi_{384,29}, \phi_{384,32}\right)$

- type $\alpha_{1^{3}}(3):\left(\phi_{729,10}, \phi_{729,12}, \phi_{729,14}\right),\left(\phi_{729,24}, \phi_{729,26}, \phi_{729,28}\right)$,

- Autres familles :

\begin{tabular}{|c|c|c|c|c|c|c|c|c|c|c|c|c|}
\hline$\chi$ & $\bar{\chi} \otimes \epsilon$ & $f_{\chi}$ & \multicolumn{3}{|c|}{$p=2$} & \multicolumn{3}{|c|}{$p=3$} & \multicolumn{4}{|c|}{$c$} \\
\hline $\bar{\phi}_{105,20}$ & $\phi_{105,26}$ & -6 & 1 & & & 1 & & & 1 & & & \\
\hline$\phi_{105,22}$ & $\phi_{105,28}$ & -6 & 1 & & & 1 & & & 1 & & & \\
\hline 189,18 & $\phi_{189,24}$ & 6 & & 1 & & & 1 & & & 1 & & \\
\hline 315,18 & $\phi_{315,24}$ & 6 & & 1 & & & 1 & & & & 1 & \\
\hline 336,17 & $\phi_{336,25}$ & $-6 \zeta_{3}^{2}$ & & 1 & & & & & 1 & & & 1 \\
\hline$\phi_{336,19}$ & $\phi_{336,23}$ & $-6 \zeta_{3}$ & & 1 & & & & & 1 & & & 1 \\
\hline$\phi_{420,14}$ & $\phi_{420,22}$ & $6 \zeta_{3}^{2}$ & & 1 & & 1 & 1 & & 1 & & 1 & \\
\hline$\phi_{420,16}$ & $\phi_{420,20}$ & $6 \zeta_{3}$ & & & 1 & 1 & 1 & & 1 & & 1 & \\
\hline$\phi_{504,15}$ & $\phi_{504,21}$ & 6 & & 11 & & & & 1 & & 1 & 1 & \\
\hline$\phi_{756,14}$ & $\phi_{756,22}$ & $-6 \zeta_{3}^{2}$ & & $\begin{array}{ll}1 & 1\end{array}$ & & & & 1 & 2 & & 1 & \\
\hline$\phi_{756,16}$ & $\phi_{756,20}$ & $-6 \zeta_{3}$ & & & 1 & & & 1 & 2 & & 1 & \\
\hline$\phi_{840,13}^{\prime \prime}$ & $\phi_{840,19}$ & 6 & & & 1 & & & 1 & 1 & 1 & 1 & 1 \\
\hline$\phi_{840,17}$ & $\phi_{840,23}^{\prime}$ & 6 & & & 1 & & & 1 & 1 & 1 & 1 & 1 \\
\hline$\phi_{945,14}$ & $\phi_{945,20}$ & 6 & 1 & & 1 & & 1 & 1 & 2 & 1 & 1 & 1 \\
\hline 945,16 & $\phi_{945,22}$ & 6 & 1 & & 1 & & 1 & 1 & 2 & 1 & 1 & 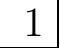 \\
\hline
\end{tabular}

\begin{tabular}{|c|c|c|c|c|c|c|c|c|}
\hline$\chi$ & $f_{\chi}$ & \multicolumn{2}{|c|}{$p=2$} & $p=3$ & & \multicolumn{2}{|c|}{$p=7$} & $c$ \\
\hline $\bar{\phi}_{20,33}^{\prime}$ & 42 & 1 & & 1 & & 1 & & \\
\hline$\phi_{20,33}^{\prime \prime}$ & 42 & 1 & & 1 & & 1 & & \\
\hline$\phi_{120,21}^{\prime}$ & 7 & & & & & & 1 & \\
\hline$\phi_{120,21}^{\prime \prime}$ & 7 & & & & & & 1 & \\
\hline$\phi_{540,17}$ & 6 & 1 & & 21 & & & & 1 \\
\hline$\phi_{540,19}$ & 6 & 1 & & 21 & & & & 1 \\
\hline$\phi_{540,21}^{\prime}$ & -42 & 2 & & 21 & & 1 & 1 & 1 \\
\hline$\phi_{540,21}^{\prime \prime \prime}$ & -42 & 2 & 1 & 21 & & & & 1 \\
\hline$\phi_{560,18}^{\prime \prime}$ & 6 & 12 & & 1 & & & & 1 \\
\hline$\phi_{560,18}^{\prime \prime \prime}$ & 6 & 21 & 1 & 1 & & & & 1 \\
\hline$\phi_{720,16}$ & 6 & & 1 & 1 & & & & 1 \\
\hline$\phi_{720,20}$ & 6 & & 1 & 1 & & & & 1 \\
\hline$\phi_{1260,17}$ & 6 & 1 & 1 & 12 & & & & 2 \\
\hline$\phi_{1260,19}$ & 6 & 1 & 1 & 12 & & & & ת \\
\hline$\phi_{1280,18}$ & 6 & & 1 & 11 & & & & 2 \\
\hline$\phi_{1280,15}$ & 42 & & 1 & 22 & & & 211 & 2 \\
\hline & & & & & & & & \\
\hline
\end{tabular}

Pour justifier ces résultats, il nous faut démontrer que la réduction modulo 3 de $\phi_{420,11}$ reste simple. Soit $C$ le bloc de $\mathcal{O}_{\mathfrak{p}} \mathcal{H}$ contenant ce caractère.

Soit $W^{\prime}$ un sous-groupe parabolique de type $G_{33}$. Soit $L$ un $k_{\mathfrak{p}} \mathcal{O}\left(W^{\prime}\right)$ module simple, de caractère $d\left(\phi_{10,8}^{\prime}\right)$. Soit $C^{\prime}$ le bloc de $\mathcal{O}_{\mathfrak{p}} \mathcal{H}\left(W^{\prime}\right)$ contenant $\phi_{10,8}^{\prime}$. La projection sur $C$ de $\operatorname{Ind}_{W^{\prime}}^{W} \phi_{10,8}^{\prime}$ est $\phi_{84,17}$, dont la réduction modulo 3 est un module simple $S$. Par conséquent, $C \otimes_{C^{\prime}} L \simeq S$. Soit $T$ l'autre $C$-module simple. On a $0=\operatorname{Hom}_{C}(S, T) \simeq$ $\operatorname{Hom}_{C^{\prime}}\left(L, C^{\prime} \otimes_{C} T\right)$. Par conséquent, $C^{\prime} \otimes_{C} T$ n'a pas de sous-module isomorphe à $S$. On montre de même qu'il n'a pas de quotient isomorphe à $S$. Soit $X$ un $C$-module, 
libre sur $\mathcal{O}_{\mathfrak{p}}$, de caractère $\phi_{420,11}$. Supposons sa réduction modulo $\mathfrak{p}$ non simple : elle a alors deux facteurs de composition $S$ et $T$. Alors, $C^{\prime} \otimes_{C} T$ a deux facteurs de composition distincts, $L$ et $L^{\prime}$. Mais un tel module a nécessairement un sous-module ou un quotient isomorphe à $L$, ce qui est impossible.

On montre de même que la reduction modulo 3 de $\phi_{420,35}$ est simple.

3.18. $W=G_{35}=W\left(E_{6}\right)$. Le groupe de réflexions $G_{35}$ a 17 familles de caractères, des types suivants (voir [13]) :

- type $\mathcal{M}\left(Z_{2}\right):\left(\phi_{30,3}, \phi_{15,5}, \phi_{15,4}\right),\left(\phi_{30,15}, \phi_{15,17}, \phi_{15,16}\right)$.

- type $\mathcal{M}\left(S_{3}\right):\left(\phi_{10,9}, \phi_{20,10}, \phi_{60,8}, \phi_{80,7}, \phi_{90,8}\right)$

Tous les autres familles sont de défaut 0 .

3.19. $W=G_{36}=W\left(E_{7}\right)$. Le groupe de réflexions $G_{36}$ a 35 familles de caractères, des types suivants (voir [13]) :

- type $\alpha_{1^{2}}\left(2^{2}\right):\left(\phi_{512,11}, \phi_{512,12}\right)$

- type $\mathcal{M}\left(Z_{2}\right):\left(\phi_{56,3}, \phi_{35,4}, \phi_{21,6}\right),\left(\phi_{405,8}, \phi_{216,9}, \phi_{189,10}\right),\left(\phi_{420,10}, \phi_{84,12}, \phi_{336,11}\right)$, $\left(\phi_{420,13}, \phi_{84,15}, \phi_{336,14}\right),\left(\phi_{405,15}, \phi_{216,16}, \phi_{189,17}\right),\left(\phi_{56,30}, \phi_{35,31}, \phi_{21,33}\right)$

- type $\mathcal{M}\left(S_{3}\right):\left(\phi_{70,9}, \phi_{35,13}, \phi_{280,8}, \phi_{315,7}, \phi_{280,9}\right),\left(\phi_{70,18}, \phi_{35,22}, \phi_{280,17}, \phi_{315,16}, \phi_{280,18}\right)$

Toutes les autres familles sont de défaut 0 .

3.20. $W=G_{37}=W\left(E_{8}\right)$. Le groupe de réflexions $G_{37}$ a 46 familles de caractères. Gyoja a montré que 23 d'entre elles correspondent à des blocs de défaut 0 , seize sont de type $\mathcal{M}\left(Z_{2}\right)$.

- type $\alpha_{1^{2}}\left(2^{2}\right)$ : $\left(\phi_{4096,11}, \phi_{4096,12}\right),\left(\phi_{4096,26}, \phi_{4096,27}\right)$

- type $\mathcal{M}\left(S_{3}\right):\left(\phi_{448,9}, \phi_{56,19}, \phi_{1344,8}, \phi_{1400,7}, \phi_{1008,9}\right),\left(\phi_{175,12}, \phi_{350,14}, \phi_{1050,10}, \phi_{1400,8}\right.$, $\left.\phi_{1575,10}\right),\left(\phi_{175,36}, \phi_{350,38}, \phi_{1050,34}, \phi_{1400,32}, \phi_{1575,34}\right),\left(\phi_{448,39}, \phi_{56,49}, \phi_{1344,38}, \phi_{1400,37}, \phi_{1008,39}\right)$

La dernière famille à 17 éléments a la matrice de décomposition suivante :

\begin{tabular}{|c|c|c|c|c|c|c|c|c|c|c|c|}
\hline$\phi$ & $f_{\chi}$ & \multicolumn{4}{|c|}{$p=2$} & \multicolumn{3}{|c|}{$p=3$} & \multicolumn{3}{|c|}{$p=5$} \\
\hline $\bar{\phi}_{70,32}$ & 30 & 1 & & & & 1 & & & [t & & \\
\hline$\phi_{168,24}$ & 8 & 1 & & & & & & & & & \\
\hline$\phi_{420,20}$ & 5 & & L & & & & & & & 1 & \\
\hline$\phi_{448,25}$ & 12 & & 1 & & & 1 & & & & & \\
\hline$\phi_{1134,20}$ & 6 & & & & & & 1 & & & & \\
\hline$\phi_{1344,19}$ & 4 & & & 1 & & & & & & & \\
\hline$\phi_{1400,20}$ & 24 & 1 & & 1 & & 1 & 1 & & & & \\
\hline$\phi_{1680,22}$ & 20 & & 1 & & & & & & & 1 & \\
\hline$\phi_{2016,19}$ & 6 & & & & 1 & & 1 & & & & \\
\hline$\phi_{2688,20}$ & 8 & & & & 1 & & & & & & \\
\hline$\phi_{3150,18}$ & 6 & & & & 1 & & & 1 & & & \\
\hline$\phi_{4200,18}$ & 8 & 1 & & 1 & 1 & & & & & & \\
\hline$\phi_{4480,16}$ & 120 & & 1 & 1 & 1 & & 1 & 1 & & 1 & 1 \\
\hline$\phi_{4536,18}$ & 24 & 1 & 1 & & 1 & & & 1 & & & \\
\hline$\phi_{5600,19}$ & 6 & & & & & 1 & & & & & \\
\hline$\phi_{5670,18}$ & 30 & 1 & & & & & 1 & 1 & & & 1 \\
\hline$\phi_{7168,17}$ & 12 & & 1 & 1 & 2 & & 1 & . & & & \\
\hline
\end{tabular}

Pour les sept caractères constructibles, voir Lusztig [14]. 
3.21. Groupes diédraux. Dans cette partie, nous déterminons les blocs pour les groupes diédraux $I_{2}(n), n \geq 3$. Les caractères des groupes diédraux se répartissent en trois familles : deux familles à un élément, consistant en le caractère trivial et le caractère signe, et une famille contenant les autres caractères. Les éléments de Schur pour les groupes de réflexions diédraux ont été déterminés par Kilmoyer et Solomon (cf. par exemple [11, Thm. 8.3.4]). Ce résultat a aussi été obtenu par Broué et Kim [3] et il résulte aussi de [22] dont les méthodes restent valables pour les groupes diédraux (car on dispose encore de l'algèbre $J$ de Lusztig). La preuve que nous donnons ici est beaucoup plus directe.

Théorème 3.1. Soit $W=I_{2}(n), n \geq 3$. Alors, les blocs de $\mathcal{O H}(W)$ coïncident avec les familles de Lusztig.

Preuve. Pour $\chi$ le caractère trivial ou le caractère signe, on a $c_{\chi} \in \mathcal{O}^{\times}$. D'après le lemme 2.6(b), il suffit donc de prouver que les autres caractères sont dans un même bloc.

Supposons tout d'abord que $n=2 m+1$ avec $m$ entier. Soit $\zeta$ une racine primitive $n$-ème de l'unité. Hormis les caractères triviaux et signes, il y a $m$ caractères $\rho_{i}$, $1 \leq i \leq m$, tous de degré 2 . On a $f_{\rho_{i}}^{-1}=a_{i}$, où

$$
a_{i}=\frac{\left(1-\zeta^{i}\right)\left(1-\zeta^{-i}\right)}{n} .
$$

Par conséquent, $\left|a_{i}\right|<1$ et $\sum_{i=1}^{m} a_{i}=1$. L'induite de la représentation triviale à partir d'un sous-groupe parabolique d'ordre 2 fournit un caractère constructible dans lequel tous les $\rho_{i}$ apparaissent avec multiplicité 1 . Soit $I$ un sous-ensemble propre non vide de $\{1, \ldots, m\}$ et $a_{I}=\sum_{i \in I} a_{i}$. Alors, $\left|a_{I}\right|<1$ et le module d'un conjugué galoisien de $a_{I}$ est aussi strictement inférieur à 1 (c'est encore une somme partielle de $\left.a_{i}\right)$. En particulier, la $\mathbb{Q}(\zeta) / \mathbb{Q}$-norme de $a_{I}$ n'est pas entière, donc $a_{I}$ n'est pas un entier. Par conséquent, $\sum_{i} \rho_{i}$ est un caractère projectif indécomposable, d'après le lemme 2.6(a).

Supposons maintenant $n=2 m$ avec $m$ entier et soit $\zeta$ une racine primitive $n$-ème de l'unité. Les caractères différents des caractères triviaux et signes consistent en deux caractères linéaires (avec $f_{\chi}^{-1}=2 / n$ ) et $m-1$ caractères $\rho_{i}, 1 \leq i \leq m-1$, de degré 2 (avec $f_{\rho_{i}}^{-1}=a_{i}$ ).

En induisant les deux caractères d'un sous-groupe parabolique d'ordre 2, on obtient deux caractères projectifs contenant chacun l'un des deux caractères de degré 1 et contenant les $\rho_{i}$ avec multiplicité 1 . Comme précédemment, on voit que ce sont des caractères projectifs indécomposables. On en déduit la coïncidence entre blocs de $\mathcal{O H}$ et familles.

Dans [22], ce résultat est établi pour les groupes de Weyl ou plus généralement lorsqu'on dispose de l'algèbre $J$ de Lusztig, ce qui est connu sauf pour le type $H_{4}$.

Corollaire 3.2. Soit $W$ un groupe de Coxeter fini. Alors, les blocs de l'algèbre de Hecke de $W$ sur $\mathcal{O}$ coïncident avec les familles de Lusztig. 


\section{Quelques GROUPeS NON SPETSiauX}

Dans cette partie, nous déterminons les matrices de décomposition et la division en familles pour quelques groupes de réflexions complexes exceptionnels non spetsiaux. Nous utiliserons plus tard ces résultats pour montrer que certaines propriétés des familles des groupes spetsiaux ne s'étendent pas au cas non spetsial. Les techniques développées au $\S 3$ s'appliquent sans changement au cas non spetsial. Il serait intéressant de déterminer les matrices de décomposition pour tous les groupes non spetsiaux et tous les choix de paramètres.

4.1. $W=G_{5}$. Le groupe de réflexions complexe $G_{5}$ est d'ordre 72 et possède 21 caractères irréducibles. Les matrices de décomposition de $\mathcal{O}_{\mathfrak{p}} \mathcal{H}(W)$ pour les mauvais nombres premiers 2 et 3 et la division en familles sont les suivantes :

- défaut $0:\left(\phi_{1,0}\right)$

- type $\alpha_{1^{2}}(3):\left(\phi_{2,5}^{\prime}, \phi_{2,7}^{\prime}\right),\left(\phi_{2,5}^{\prime \prime \prime}, \phi_{2,7}^{\prime \prime}\right)$

- Autres familles :

\begin{tabular}{l|l|l|l|} 
& $f_{\chi}$ & $p=3$ & $c$ \\
\hline$\phi_{3,6}$ & 3 & 1 & 1 \\
$\phi_{3,2}$ & $3 \zeta_{3}$ & 1 & 1 \\
$\phi_{3,4}$ & $3 \zeta_{3}^{2}$ & 1 & 1 \\
\hline
\end{tabular}

\begin{tabular}{|c|c|c|c|c|c|c|}
\hline & $f_{\chi}$ & \multicolumn{3}{|c|}{$p=2$} & $p=3$ & $c$ \\
\hline$\overline{\phi_{1.8}^{\prime \prime}}$ & $3 \zeta_{3}^{2}$ & 1 & & & 1 & 1 \\
\hline$\phi_{1,12}^{\prime}$ & 6 & & 1 & & 1 & 1 \\
\hline$\phi_{1,12}^{\prime \prime}$ & 6 & & & 1 & 1 & 1 \\
\hline$\phi_{1,16}$ & $3 \zeta_{3}$ & & & & 1 & 1 \\
\hline$\phi_{2,9}$ & 2 & & 1 & 1 & 1 & 2 \\
\hline
\end{tabular}

\begin{tabular}{|c|c|c|c|c|c|c|c|c|c|c|c|}
\hline & $f$ & & \multicolumn{5}{|c|}{$p=2$} & \multicolumn{2}{|c|}{$p=3$} & \multicolumn{2}{|c|}{$c$} \\
\hline$\overline{\phi_{14}^{\prime}}$ & $2\left(\zeta_{2}^{2}-\zeta_{3}\right)$ & 1 & & & & & & 1 & & 1 & \\
\hline$\phi_{1,4}^{\prime \prime 4}$ & $2\left(\zeta_{3}^{2}-\zeta_{3}\right)$ & & 1 & & & & & & 1 & & 1 \\
\hline$\phi_{1,8}^{\prime}$ & $2\left(\zeta_{3}-\zeta_{3}^{2}\right)$ & & & 1 & & & & 1 & & 1 & \\
\hline$\phi_{1,8}^{\prime \prime \prime}$ & $2\left(\zeta_{3}-\zeta_{3}^{2}\right)$ & & & & 1 & & & & 1 & & 1 \\
\hline$\phi_{2,5}^{\prime \prime}$ & 6 & & & 1 & 1 & & & 1 & 1 & 1 & 1 \\
\hline$\phi_{2,3}^{\prime}$ & 3 & & & & & 1 & & 1 & 1 & 1 & 1 \\
\hline$\phi_{2,3}^{\prime \prime \prime}$ & 3 & & & & & & L & 1 & 1 & 1 & 1 \\
\hline$\phi_{2,1}$ & 6 & 1 & 1 & & & & & 1 & 1 & 1 & 1 \\
\hline
\end{tabular}

4.2. $W=G_{12}$. Le groupe de réflexions complexe non spetsial $G_{12}$ a les cinq familles suivantes :

- défaut 0 : $\left(\phi_{1,0}\right),\left(\phi_{3,2}\right),\left(\phi_{3,6}\right),\left(\phi_{1,12}\right)$

- Autre famille :

\begin{tabular}{|c|c|c|c|c|}
\hline & $f_{x}$ & $p=2$ & $p=3$ & $c$ \\
\hline$\phi_{2,1}$ & -12 & 1 & & 1 \\
\hline$\phi_{2,4}$ & 2 & 1 & 1 & 1 \\
\hline$\phi_{2,5}$ & -12 & 1 & 1 & 1 \\
\hline$\phi_{4,3}$ & 2 & 1 & 2 & 2 \\
\hline$? ?$ & & & $*$ & \\
\hline
\end{tabular}


4.3. $W=G_{13}$. Le groupe de réflexions complexe non spetsial $G_{13}$ a les sept familles suivantes :

- défaut 0 : $\left(\phi_{1,0}\right),\left(\phi_{3,4}\right),\left(\phi_{3,6}\right),\left(\phi_{1,18}\right)$

- type $\mathcal{M}\left(Z_{2}\right):\left(\phi_{1,6}, \phi_{2,4}, \phi_{3,2}\right),\left(\phi_{1,12}, \phi_{2,10}, \phi_{3,8}\right)$

- Autre famille :

\begin{tabular}{|c|c|c|c|c|}
\hline & $f_{\chi}$ & $p=2$ & $p=3$ & $c$ \\
\hline$\overline{\phi_{2,7}^{\prime}}$ & $-12 \zeta_{4}$ & 1 & 1 & 1 \\
\hline$\phi_{2,1}$ & $12 \zeta_{4}$ & 1 & 1 & 1 \\
\hline$\phi_{2,5}$ & $12 \zeta_{4}$ & 1 & 1 & 1 \\
\hline$\phi_{2,7}^{\prime \prime}$ & $-12 \zeta_{4}$ & 1 & 1 & 1 \\
\hline$\phi_{4,3}$ & $-6 \zeta_{4}$ & 2 & 2 & 2 \\
\hline$\phi_{4,5}$ & $6 \zeta_{4}$ & 2 & 2 & 2 \\
\hline 20 & & $*$ & $* \quad *$ & \\
\hline
\end{tabular}

\section{Quelques COnsÉquences}

Les résultats de la partie 3, joints au résultat du second auteur 22] pour les groupes de Weyl et aux travaux de Broué et Kim [3] sur les groupes imprimitifs (séries infinies $G(d, 1, n)$ et $G(d, d, n))$ complètent la détermination des familles de tous les groupes spetsiaux.

Nous déduisons de cette description des propriétés générales, satisfaites par toutes les familles de groupes de réflexions spetsiaux — nous ne possèdons pour le moment aucune preuve directe de ces propriétés.

Soit $W$ un groupe de réflexions complexe, de corps de définition un corps de nombre $k$. Soit $\mu(k)$ l'ordre du groupe des racines de l'unité de $k$ et $y$ tel que $y^{\mu(k)}=x$. Alors, sous l'hypothèse que la conjecture 2.1 est vraie, le résultat principal de [20] montre que l'algèbre $K \mathcal{H}(W)$ est déployée pour $K=k(y)$. Par conséquent, les éléments de Schur $c_{\chi}(\chi \in \operatorname{Irr}(W))$ sont contenus dans $K$.

Pour $\chi \in \operatorname{Irr}(W)$, soit $\delta_{\chi}=P(W) / c_{\chi}$ le degré générique de $\chi$, une fonction rationelle de $y$. Soient $a(\chi) \in \frac{1}{\mu(k)} \mathbb{Z}$ le plus grand nombre tel que $y^{-a(\chi) \mu(k)} \delta_{\chi}$ est un polynôme en $y$ et $A(\chi) \in \frac{1}{\mu(k)} \mathbb{Z}$ le plus petit nombre tel que $y^{A(\chi) \mu(k)} \delta_{\chi}\left(y^{-1}\right)$ est un polynôme en $y$.

Par définition, si de plus $W$ est spetsial, les $c_{\chi}$ sont même rationnels, c'est-à-dire, contenus dans $k(x)$, et alors $a(\chi), A(\chi) \in \mathbb{Z}$.

D'après Broué-Malle-Michel [7, Cor. 6.9], on a

$$
a(\chi)+A(\chi)=\frac{N(\chi)+N\left(\chi^{*}\right)}{\chi(1)},
$$

donc, $a(\chi)+A(\chi)$ est constant sur les familles par le lemme 2.8. Les résultats explicites précédents démontrent le suivant, bien connu pour les groupes de Coxeter (voir Lusztig [14]), et pour les groupes de réflexions imprimitifs (voir Broué-Kim [3, Prop. 4.5]) :

Théorème 5.1. Soit $W$ un groupe de réflexions complexe spetsial. Supposons les conjectures 2.1, 2.9 et 2.9 vraies si $W$ est de type exceptionnel. Alors a $(\chi)($ et $A(\chi))$ est constant sur toute famille de $W$.

Notons que nous ne connaissons pas de contre-exemple au théorème 5.1 pour des groupes non spetsiaux. 
Le théorème 5.1 ne se déduit pas du lemme 2.8 qui affirme la constance de $a+A$, comme le montre l'exemple suivant :

Exemple 5.2. L'ensembles des caractères du groupe $G_{26}$ avec $a+A=30$ est la réunion de deux familles, l'une avec $a(\mathcal{F})=4$ et l'autre avec $a(\mathcal{F})=5$.

Le théorème 5.1 permet de définir $a(\mathcal{F})$ pour une famille $\mathcal{F}$ comme valeur commune des $a(\chi)$ pour $\chi \in \mathcal{F}$. De même, nous posons $A(\mathcal{F})=A(\chi)$ pour $\chi \in \mathcal{F}$.

Un caractère $\chi \in \operatorname{Irr}(W)$ est dit spécial si

$$
a(\chi)=b(\chi) \text {, }
$$

où, comme noté dans $3.3, b(\chi)$ est le plus grand entier tel que $x^{-b(\chi)} R_{\chi}$ est un polynôme. Les caractères spéciaux ont été determinés dans [21, 8B].

Théorème 5.3. Soit $W$ un groupe de réflexions complexe spetsial. Supposons les conjectures 2.1, 2.9 et 2.9 vraies si $W$ est de type exceptionnel. Alors chaque famille contient un unique caractère spécial.

Pour les séries infinies, cela découle de [17, Bem. 2.24 et Lemma 5.16] et du fait que les familles combinatoires coïncident avec les blocs [3, Thm. 3.16]. Ce résultat a été vérifé (cas par cas) pour les groupes de Coxeter par Lusztig [14].

La proposition suivante montre que pour $W$ spetsial les caractères spéciaux peuvent aussi être définis comme les caractères $\chi$ tels que $b(\chi)=a(\mathcal{F}(\chi))$.

D'après [21, Prop. 8.1], on a :

Proposition 5.4. Soient $W$ un groupe de réflexions complexe spetsial et $\mathcal{F}$ une famille de $W$. Alors

$$
a(\mathcal{F}) \leq b(\chi) \quad \text { et } \quad B\left(\chi^{*}\right) \leq A(\mathcal{F})
$$

pour tous $\chi \in \mathcal{F}$. De plus, les inégalités sont strictes sauf si $\chi$ est le caractère spécial de $\mathcal{F}$.

Pour les groupes non spetsiaux, les assertions 5.3 et 5.4 deviennent fausses :

Exemple 5.5. Les familles de $W=G_{5}$ sont décrites dans 4.1. La fonction $a$ est constante sur les familles. Pour les deux familles de type $\alpha_{1^{2}}(3)$, on a $a(\mathcal{F})=4$, mais ces familles ne contiennent pas de caractère spécial; de même pour les deux familles à 3 et 5 caractères. De plus, pour la famille à 3 caractères, on a $a(\mathcal{F})=8 / 3>2=$ $b\left(\phi_{3,2}\right)$.

De même, pour la famille $\left(\phi_{3,2}\right)$ de $G_{12}$, on a $a(\mathcal{F})=1$.

Notons aussi que :

Proposition 5.6. Soit $W$ un groupe de réflexions complexe spetsial. Supposons les conjectures 2.1, 2.2 et 2.3 vraies si $W$ est de type exceptionnel. Alors toutes les familles de $\operatorname{Irr}(W)$ sont invariantes par les automorphismes galoisiens de $\operatorname{Irr}(W)$.

Remarque 5.7. Soit $W$ un groupe spetsial, $\phi$ un caractère constructible de $W$ dans la famille $\mathcal{F}$ et $\chi_{s}$ le caractère spécial de $\mathcal{F}$. Alors,

$$
\left\langle\phi, \chi_{s}-\sum_{\chi \in \mathcal{F}} \frac{1}{f_{\chi}} \chi\right\rangle=0
$$


lorsque $W$ est exceptionnel. Cette propriété est aussi vraie lorsque $W$ est un groupe de Weyl : elle résulte de l'invariance des caractères constructibles par transformation de Fourier, elle-même conséquence de la commutation entre la transformation de Fourier et la $J$-induction. Il est probable que cette propriété est vraie pour les groupes spetsiaux de la série infinie. Il est aussi envisageable que cette propriété soit vraie plus généralement lorsque $\phi$ est le caractère d'un $\mathcal{O H}$-module projectif.

Notons aussi que lorsque $\sum_{\chi \in \mathcal{F}} \frac{\left\langle\phi_{1}, \chi\right\rangle}{f_{\chi}} \notin \mathcal{O}$ pour tout sous-caractère propre $\phi_{1}$ de $\phi$, alors $\phi$ est le caractère d'un $\mathcal{O H}$-module projectif indécomposable.

Dans le cas des groupes de Coxeter finis, on a $\left\langle\phi, \chi_{s}\right\rangle=1$. Dans les cas étudiés dans 3, il arrive que cette multiplicité soit $>1$. Ceci suggère que notre définition de caractères constructibles n'est peut-être pas la bonne dans ces cas.

\section{Compatibilité entre familles, $\mathcal{O}$-Blocs et $d$-SÉRies De HARISH-CHANDRA}

Les $\mathcal{O}$-blocs d'algèbres de Hecke peuvent être considérés pour d'autres choix de paramètres. Dans cette partie, nous considérons un cas particulièrement important : celui des algèbres de Hecke associées aux "groupes de Weyl" relatifs d'une $d$-série de Harish-Chandra de caractères unipotents dans un groupe réductif fini. Nous établissons alors une compatibilité avec les familles de Lusztig.

On considère un groupe réductif connexe sur une clôture algébrique d'un corps fini, muni d'un endomorphisme dont une puissance est un Frobenius et on note $G$ le groupe des points fixes par cet endomorphisme (un groupe fini "de type de Lie"). On note $\mathcal{E}(G)$ l'ensemble de ses caractères unipotents. Soit $d>1$ un entier tel que le polynôme cyclotomique $\Phi_{d}(x)$ divise l'ordre générique de $G$. Suivant Broué-MalleMichel [6, Thm. 3.2], on considère la partition de $\mathcal{E}(G)$ en $d$-séries de Harish-Chandra (cf. aussi Broué-Malle [5])

$$
\mathcal{E}(G)=\coprod_{L, \lambda} \mathcal{E}(G,(L, \lambda))
$$

de $\mathcal{E}(G)$, où $L$ décrit l'ensemble des sous-groupes de Levi $d$-ployés de $G$ à conjugaison près, $\lambda$ décrit l'ensemble des caractères $d$-cuspidaux de $L$, et $\mathcal{E}(G,(L, \lambda))$ consiste des constituants de $R_{L}^{G}(\lambda)$.

En outre, pour chaque paire cuspidale $(L, \lambda)$, on a une bijection entre $\mathcal{E}(G,(L, \lambda))$ et l'ensemble des caractères irréductibles de l'algèbre de Hecke $\mathcal{H}(G,(L, \lambda))$ du groupe de Weyl relatif $W_{G}(L, \lambda)$, avec certains paramètres. Ceci est décrit explicitement dans [17, Satz 3.14 et 6.10] pour les groupes classiques et dans [4] pour les groupes exceptionnels. D'après Broué-Malle-Michel [6, Thm. 5.24], la partition (3) est identique à la partition de $\mathcal{E}(G)$ en $\ell$-blocs, pour tout nombre premier $\ell$ divisant $\Phi_{d}(q)$ mais ne divisant pas l'ordre du groupe de Weyl de $G$.

La partition en $\mathcal{O}$-blocs (cf. chapitre 2) des caractères irréductibles de chaque algèbre de Hecke relative $\mathcal{H}(G,(L, \lambda))$, fournit une partition

$$
\mathcal{E}(G,(L, \lambda))=\coprod_{\mathcal{G}} \mathcal{G} .
$$

On a aussi une décomposition en familles de Lusztig de $\mathcal{E}(G)$ [15, Chap. 4]. Pour les caractères unipotents de la série principale ( $d=1$ et $L$ est un tore), elle coïncide avec la partition précédente en $\mathcal{O}$-blocs [22]. 
Ce résultat se généralise au cas des paires cuspidales quelconques et à tout $d$ en le théorème suivant :

Théorème 6.1. Soit $G$ un groupe réductif connexe sur un corps fini et $\mathcal{E}(G,(L, \lambda))$ une d-série de Harish-Chandra de $\mathcal{E}(G)$ d'algèbre de Hecke relative $\mathcal{H}(G,(L, \lambda))$. Supposons les conjectures 2.1, 2.7 et 2.9 vraies si le groupe de Weyl relative $W_{G}(L, \lambda)$ est de type exceptionnel non-réel. Alors, les deux partitions suivantes de $\mathcal{E}(G,(L, \lambda))$ coincident :

$$
\coprod_{\mathcal{F}}(\mathcal{F} \cap \mathcal{E}(G,(L, \lambda)))=\coprod_{\mathcal{G}} \mathcal{G}
$$

où $\mathcal{F}$ décrit les familles de Lusztig de $\mathcal{E}(G)$ et $\mathcal{G}$ décrit les $\mathcal{O}$-blocs de $\operatorname{Irr}(\mathcal{H}(G,(L, \lambda)))$. En d'autres termes, les $\mathcal{O}$-blocs de $\operatorname{Irr}(\mathcal{H}(G,(L, \lambda)))$ sont les intersections des familles de Lusztig avec $\mathcal{E}(G,(L, \lambda))$.

Réciproquement, la partition en familles de Lusztig de $\mathcal{E}(G)$ est la plus fine partition telle que la propriété précédente soit vraie pour tout d et toute paire d-cuspidale $(L, \lambda)$.

Preuve. Le reste de cette partie est consacré à la preuve de ce théorème. Notons tout d'abord qu'il suffit de considérer le cas où le groupe algébrique est simple.

Considérons pour commencer le cas des groupes classiques. Dans les groupes de type $A_{n}$ et ${ }^{2} A_{n}$, toutes les familles sont réduites à des singletons, tout comme les blocs des algèbres de Hecke relatives sur $\mathcal{O}$.

Les caractères unipotents des autres groupes classiques sont paramétrés par des symboles. Un symbole $\Lambda=\{S, T\}$ est la donnée de deux ensembles finis $S$ et $T$ d'entiers positifs. On dit que deux symboles sont équivalents si l'un peut être obtenu à partir de l'autre par une suite d'opérations de décalage

$$
\{S, T\} \mapsto\{\{0\} \cup S+1,\{0\} \cup T+1\}
$$

Le défaut d'un symbole est $\operatorname{def}(S, T)=|| S|-| T||$. Le rang de $\Lambda=(S, T)$ est

$$
\sum_{\lambda \in \Lambda} \lambda-\left\lfloor\frac{(|\Lambda|-1)^{2}}{4}\right\rfloor .
$$

Pour $G$ de type $B_{n}$ ou $C_{n}$, alors $\mathcal{E}(G)$ est paramétré par les classes d'équivalence de symboles de rang $n$ et de défaut impair. Deux caractères unipotents sont dans la même famille de Lusztig si et seulement si les multi-ensembles réunions de $S$ et $T$ coïncident.

Le paramétrage des séries de Harish-Chandra fourni par [17, Satz 3.14] et la description des blocs pour les algèbres de Hecke donnée par Broué-Kim [3, Thm. 3.16] montrent que la première partie du théorème est vraie.

Passons à la deuxième partie du théorème. Nous dirons qu'un sous-ensemble de $\mathcal{E}(G)$ est une famille potentielle si elle est un sous-ensemble minimal avec la propriété que son intersection avec toute $d$-série de Harish-Chandra est une union de $\mathcal{O}$-blocs pour l'algèbre de Hecke relative correspondante. Il s'agit donc de démontrer que les familles potentielles sont les familles. Notons pour commencer que la $d$-série de Harish-Chandra d'un symbole est déterminée par le $d$-cœur du symbole lorsque $d$ est impair et par le $d / 2$-cocœur du symbole lorsque $d$ est pair [6, Section 3A]. En particulier, la 1-série de Harish-Chandra d'un symbole est déterminée par le défaut du symbole. On en déduit que l'intersection d'une famille potentielle avec une famille de Lusztig est une union de parties de la partition de cette famille suivant les défauts 
des symboles. Il nous reste donc à démontrer qu'étant donnés deux défauts $t_{1}$ et $t_{2}$ apparaissant dans une famille de Lusztig donnée, alors il existe un défaut $d$ et deux symboles $\Lambda_{1}, \Lambda_{2}$ de défauts $\operatorname{def}\left(\Lambda_{i}\right)=t_{i}$, tels que $\Lambda_{1}$ et $\Lambda_{2}$ sont dans la même $d$-série de Harish-Chandra, c'est-à-dire, ont le même $d$-cœur (respectivement $d / 2$-cocœur).

Soit donc $\mathcal{F}$ une famille de Lusztig avec au moins deux éléments. Le multi-ensemble des éléments de tout symbole de $\mathcal{F}$ contient au moins trois singletons. Soit $\Lambda_{1}=$ $\{S, T\} \in \mathcal{F}$. On peut supposer qu'il existe deux singletons $\lambda_{1}, \lambda_{2}$ dans $S$ avec $\lambda_{1}<$ $\lambda_{2}$. Soit $\Lambda^{\prime}$ le symbole obtenu à partir de $\Lambda_{1}$ en enlevant un cocrochet de longueur $l:=\lambda_{2}-\lambda_{1}$ à la position $\lambda_{2}$ de $S$ et soit $\Lambda_{2}$ le symbole obtenu à partir de $\Lambda^{\prime}$ en ajoutant un cocrochet de longueur $l$ à la position $\lambda_{1}$ de $T$. Alors, $\Lambda_{2}$ est obtenu à partir de $\Lambda_{1}$ en déplaçant $\lambda_{1}$ et $\lambda_{2}$ de $S$ à $T$, donc $\Lambda_{2} \in \mathcal{F}$. Par construction, $\Lambda_{1}$ et $\Lambda_{2}$ ont le même $l$-cocœur, donc appartiennent à la même $2 l$-série de Harish-Chandra. En outre, les défauts de $\Lambda_{1}$ et $\Lambda_{2}$ diffèrent de 4 . Notons enfin que si $\mathcal{F}$ contient des symboles de défaut $t$, alors tout entier impair de $\{1, \ldots, t\}$ apparaît comme défaut d'un symbole de $\mathcal{F}$. La construction précédente permet donc d'atteindre tous les défauts de la famille.

Pour les groupes de type $D_{n}$ (respectivement ${ }^{2} D_{n}$ ), alors $\mathcal{E}(G)$ est paramétré par des symboles de défaut congru à $0(\bmod 4)($ respectivement $2(\bmod 4))$. Des arguments similaires aux précédents s'appliquent à cette situation.

Passons maintenant aux types exceptionnels. Les $d$-séries de Harish-Chandra de groupes de Weyl relatifs cycliques sont décrites dans Broué-Malle [4, Tab. 8.1 et 8.3]. On déduit facilement de cette description la première partie du thèorème, dans ce cas.

Rappelons que le cas de la 1-série de Harish-Chandra principale est déjà connu par 22.

Dans la suite nous déterminons les blocs des algèbres de Hecke associées aux groupes de réflexions $W^{\prime}$ de type exceptionnel pour les paramètres qui proviennent de cas où $W^{\prime}$ intervient comme groupe de Weyl relatif d'une $d$-série de Harish-Chandra de caractères unipotents d'un groupe exceptionnel (voir table 31) et nous démontrons la première partie du théorème 6.1 pour ces cas. Seul un groupe non cylique et non exceptionnel $W^{\prime}$ apparaît : dans ce cas, les blocs sont déterminés dans [3] et on déduit la première partie du théorème.

Nous traitons aussi le cas du groupe de Coxeter $H_{4}$.

La deuxième partie du théorème 6.1 pour les groupes $G$ exceptionnels se réduit alors à une vérification facile.

6.1. $W=G_{4}$. Le groupe $W=G_{4}$ intervient comme groupe relatif dans les groupes réductifs de type ${ }^{3} D_{4}$ (cf 专, Folg. 5.6] pour les paramètres). Dans ce cas, l'algèbre de Hecke a un seul bloc non trivial, de caractères $\left(\phi_{1,8}, \phi_{2,1}, \phi_{3,2}\right)$.

6.2. $W=G_{5}$. Le groupe $G_{5}$ intervient comme groupe relatif dans les groupes réductifs de type $F_{4}, E_{6}$ et $E_{8}$ (cf [4, Table 5.9]). Nous indiquons seulement les blocs non triviaux :

Dans $F_{4}:\left(\phi_{1,8}^{\prime}, \phi_{1,8}^{\prime \prime \prime}, \phi_{2,5}^{\prime \prime}\right),\left(\phi_{1,12}^{\prime}, \phi_{1,12}^{\prime \prime}, \phi_{2,9}\right),\left(\phi_{1,4}^{\prime}, \phi_{1,4}^{\prime \prime}, \phi_{1,16}, \phi_{2,1}, \phi_{2,7}^{\prime}, \phi_{2,7}^{\prime \prime}, \phi_{3,2}\right.$, $\left.\phi_{3,4}, \phi_{3,6}\right)$.

Dans $E_{6}:\left(\phi_{1,4}^{\prime}, \phi_{1,16}, \phi_{2,7}^{\prime \prime}\right),\left(\phi_{1,8}^{\prime}, \phi_{1,8}^{\prime \prime \prime}, \phi_{2,5}^{\prime \prime}\right),\left(\phi_{1,12}^{\prime}, \phi_{2,3}^{\prime}, \phi_{3,2}, \phi_{3,4}, \phi_{3,6}\right)$.

Dans $E_{8}:\left(\phi_{1,16}, \phi_{2,1}, \phi_{3,2}, \phi_{3,4}, \phi_{3,6}\right)$. 
TAB. 3. Groupes de Weyl relatifs non cycliques

\begin{tabular}{|c|c|c|c|c|c|c|}
\hline$G$ & $d=$ & 3 & 4 & 5 & 8 & 12 \\
\hline${ }^{3} D_{4}$ & $G(6,6,2)$ & $G_{4}$ & & & & \\
\hline${ }^{2} F_{4}$ & $G(8,8,2)$ & - & $G_{12}$ & & $G_{8}$ & \\
\hline$F_{4}$ & $G_{28}, G(2,1,2)$ & $G_{5}$ & $G_{8}$ & & & \\
\hline$E_{6}$ & $G_{35}$ & $G_{25}$ & $G_{8}$ & & & \\
\hline${ }^{2} E_{6}$ & $G_{28}$ & $G_{5}$ & $G_{8}$ & & & \\
\hline$E_{7}$ & $G_{36}, G(2,1,3)$ & $G_{26}$ & $G_{8}, G(4,1,2)$ & & & \\
\hline$E_{8}$ & $G_{37}, G_{28}, G(6,6,2)$ & $G_{32}$ & $G_{31}$ & $G_{16}$ & $G_{9}$ & $G_{10}$ \\
\hline$H_{4}$ & $G_{30}$ & $G_{20}$ & $G_{22}$ & & & \\
\hline
\end{tabular}

6.3. $W=G_{8}$. Le groupe $G_{8}$ intervient comme groupe relatif dans les groupes réductifs de type ${ }^{2} F_{4}, F_{4}, E_{6}, E_{7}$ et $E_{8}$ (cf [4, Table 5.12]). Les blocs non triviaux sont :

Dans ${ }^{2} F_{4}:\left(\phi_{2,1}, \phi_{2,4}\right),\left(\phi_{2,10}, \phi_{2,13}\right),\left(\phi_{1,6}, \phi_{1,12}, \phi_{2,7}^{\prime}, \phi_{2,7}^{\prime \prime}, \phi_{3,4}, \phi_{3,6}, \phi_{4,3}, \phi_{4,5}\right)$.

Dans $F_{4}:\left(\phi_{2,1}, \phi_{2,7}^{\prime}\right),\left(\phi_{2,7}^{\prime \prime}, \phi_{2,13}\right),\left(\phi_{1,6}, \phi_{1,18}, \phi_{2,4}, \phi_{2,10}, \phi_{3,2}, \phi_{3,6}, \phi_{4,3}, \phi_{4,5}\right)$.

Dans $E_{6}:\left(\phi_{1,6}, \phi_{2,4}, \phi_{3,2}\right),\left(\phi_{1,12}, \phi_{2,10}, \phi_{3,8}\right),\left(\phi_{2,7}^{\prime}, \phi_{2,7}^{\prime \prime}, \phi_{4,3}, \phi_{4,5}\right)$.

Dans $E_{7}:\left(\phi_{2,1}, \phi_{2,7}^{\prime}\right),\left(\phi_{2,7}^{\prime \prime}, \phi_{2,13}\right),\left(\phi_{3,2}, \phi_{3,6}\right),\left(\phi_{4,3}, \phi_{4,5}\right),\left(\phi_{1,6}, \phi_{1,18}, \phi_{2,10}\right)$.

Dans $E_{8}:$ a) $\left(\phi_{2,1}, \phi_{2,7}^{\prime}\right),\left(\phi_{2,7}^{\prime \prime}, \phi_{2,13}\right),\left(\phi_{3,2}, \phi_{3,6}\right),\left(\phi_{4,3}, \phi_{4,5}\right),\left(\phi_{1,6}, \phi_{1,18}, \phi_{2,10}\right)$;

b) $\left(\phi_{2,1}, \phi_{2,7}^{\prime \prime}\right),\left(\phi_{2,7}^{\prime}, \phi_{2,13}\right),\left(\phi_{3,4}, \phi_{3,8}\right),\left(\phi_{4,3}, \phi_{4,5}\right),\left(\phi_{1,0}, \phi_{1,12}, \phi_{2,4}\right)$;

c) $\left(\phi_{2,1}, \phi_{2,13}, \phi_{4,3}, \phi_{4,5}\right)$;

d) $\left(\phi_{2,7}^{\prime}, \phi_{2,7}^{\prime \prime}, \phi_{4,3}, \phi_{4,5}\right)$.

6.4. $W=G_{9}$. Le groupe $G_{9}$ intervient comme groupe relatif dans les groupes réductifs de type $E_{8}$ (cf [18, Table 9]). Les blocs non triviaux sont :

$\left(\phi_{2,7}^{\prime}, \phi_{2,11}^{\prime \prime}\right),\left(\phi_{2,7}^{\prime \prime}, \phi_{2,10}\right),\left(\phi_{1,12}^{\prime}, \phi_{1,12}^{\prime \prime}, \phi_{2,8}, \phi_{2,11}^{\prime}\right),\left(\phi_{1,6}, \phi_{1,30}, \phi_{2,13}, \phi_{2,17}\right)$,

$\left(\phi_{2,1}, \phi_{2,4}, \phi_{2,5}, \phi_{2,14}, \phi_{4,3}, \phi_{4,5}, \phi_{4,7}, \phi_{4,9}\right)$.

6.5. $W=G_{10}$. Le groupe $G_{10}$ intervient comme groupe relatif dans les groupes réductifs de type $E_{8}$ (voir [18, Table 9]). Les blocs non triviaux sont :

$\left(\phi_{1,6}, \phi_{1,18}\right),\left(\phi_{1,22}, \phi_{1,34}\right),\left(\phi_{2,1}, \phi_{2,7}^{\prime}\right),\left(\phi_{2,5}, \phi_{2,11}^{\prime}\right),\left(\phi_{2,9}, \phi_{2,15}^{\prime}\right),\left(\phi_{2,7}^{\prime \prime}, \phi_{2,13}\right)$,

$\left(\phi_{2,11}^{\prime \prime}, \phi_{2,17}\right),\left(\phi_{2,15}^{\prime \prime}, \phi_{2,21}\right),\left(\phi_{4,3}, \phi_{4,9}\right),\left(\phi_{4,7}, \phi_{4,13}\right)$,

$\left(\phi_{1,16}, \phi_{2,10}, \phi_{3,2}, \phi_{3,6}^{\prime \prime}, \phi_{3,10}^{\prime}\right),\left(\phi_{1,12}, \phi_{2,18}, \phi_{3,6}^{\prime}, \phi_{3,10}^{\prime \prime}, \phi_{3,14}\right)$,

$\left(\phi_{1,14}, \phi_{1,26}, \phi_{2,8}, \phi_{3,12}^{\prime}, \phi_{3,12}^{\prime \prime}, \phi_{3,8}^{\prime}, \phi_{3,16}, \phi_{3,8}^{\prime \prime}, \phi_{3,4}, \phi_{4,11}, \phi_{4,5}\right)$,

Notons que pour montrer que $\left(\phi_{2,9}, \phi_{2,15}^{\prime}\right)$ forme une famille, nous avons utilisé Chevie, pour calculer certaines valeurs de caractères de l'algèbre de Hecke.

6.6. $W=G_{12}$. Le groupe $G_{12}$ intervient comme groupe relatif dans les groupes réductifs de type ${ }^{2} F_{4}$ (cf [4, Folg. 5.14]). Il y a un seul bloc non trivial :

$\left(\phi_{2,1}, \phi_{2,4}, \phi_{2,5}, \phi_{4,3}\right)$.

6.7. $W=G_{16}$. Le groupe $G_{16}$ intervient comme groupe relatif dans les groupes réductifs de type $E_{8}$ ( $\operatorname{cf}[18$, Table 9]). Les blocs non triviaux sont :

$\left(\phi_{1,12}, \phi_{2,7}, \phi_{3,2}\right),\left(\phi_{1,36}, \phi_{2,31}, \phi_{3,26}\right),\left(\phi_{3,10}^{\prime}, \phi_{3,10}^{\prime \prime}, \phi_{6,5}\right),\left(\phi_{3,18}^{\prime}, \phi_{3,18}^{\prime \prime}, \phi_{6,13}\right)$,

$\left(\phi_{2,13}^{\prime}, \phi_{2,13}^{\prime \prime}, \phi_{4,3}, \phi_{4,8}\right),\left(\phi_{2,25}^{\prime}, \phi_{2,25}^{\prime \prime}, \phi_{4,15}, \phi_{4,20}\right),\left(\phi_{4,6}, \phi_{4,11}\right),\left(\phi_{4,12}, \phi_{4,17}\right)$,

$\left(\phi_{1,24}, \phi_{2,19}^{\prime}, \phi_{2,19}^{\prime \prime}, \phi_{3,14}^{\prime}, \phi_{3,14}^{\prime \prime}, \phi_{4,9}, \phi_{4,14}, \phi_{5,4}, \phi_{5,8}, \phi_{5,10}, \phi_{5,12}, \phi_{5,16}, \phi_{6,9}\right)$. 
6.8. $W=G_{20}$. Le groupe $G_{20}$ intervient comme groupe relatif pour le spets de type $H_{4}$ (cf [18, Table 9]). Les blocs non triviaux sont :

$\left(\phi_{2,1}, \phi_{2,7}\right),\left(\phi_{2,21}, \phi_{2,27}\right),\left(\phi_{4,3}, \phi_{4,6}\right),\left(\phi_{4,13}, \phi_{4,16}\right)$,

$\left(\phi_{1,20}, \phi_{2,11}, \phi_{2,17}, \phi_{3,2}, \phi_{3,6}, \phi_{3,10}^{\prime}, \phi_{3,10}^{\prime \prime}, \phi_{3,12}, \phi_{3,14}, \phi_{4,8}, \phi_{4,11}, \phi_{5,8}, \phi_{6,5}, \phi_{6,7}, \phi_{6,9}\right)$.

6.9. $W=G_{22}$. Le groupe $G_{22}$ intervient comme groupe relatif pour le spets de type $H_{4}$ (cf [18, Table 9]). Les blocs non triviaux sont :

$\left(\phi_{3,2}, \phi_{3,6}\right),\left(\phi_{3,12}, \phi_{3,16}\right),\left(\phi_{2,1}, \phi_{2,7}, \phi_{2,11}, \phi_{2,13}, \phi_{4,3}, \phi_{4,6}, \phi_{4,8}, \phi_{4,9}, \phi_{6,5}, \phi_{6,7}\right)$.

6.10. $W=G_{25}$. Le groupe $G_{25}$ intervient comme groupe relatif dans les groupes réductifs de type $E_{6}$ (cf [4. Folg. 5.16]). Les blocs non triviaux sont :

$\left(\phi_{3,5}^{\prime}, \phi_{3,5}^{\prime \prime}, \phi_{6,2}\right),\left(\phi_{3,13}^{\prime}, \phi_{3,13}^{\prime \prime}, \phi_{6,10}\right),\left(\phi_{1,12}, \phi_{2,9}, \phi_{3,6}, \phi_{8,6}, \phi_{9,5}, \phi_{9,7}\right)$.

6.11. $W=G_{26}$. Le groupe $G_{26}$ intervient comme groupe relatif dans les groupes réductifs de type $E_{7}$ (cf. [21, Prop. 7.1]). Les blocs non triviaux sont :

$\left(\phi_{1,12}, \phi_{2,9}, \phi_{3,6}\right),\left(\phi_{1,21}, \phi_{2,18}, \phi_{3,15}\right),\left(\phi_{3,5}^{\prime}, \phi_{3,5}^{\prime \prime}, \phi_{6,2}\right),\left(\phi_{3,16}^{\prime}, \phi_{3,16}^{\prime \prime}, \phi_{6,13}\right)$,

$\left(\phi_{3,8}^{\prime}, \phi_{3,8}^{\prime \prime}, \phi_{6,5}\right),\left(\phi_{3,13}^{\prime}, \phi_{3,13}^{\prime \prime}, \phi_{6,10}\right),\left(\phi_{8,6}^{\prime \prime}, \phi_{8,9}^{\prime}\right),\left(\phi_{1,9}, \phi_{2,15}, \phi_{8,3}, \phi_{8,6}^{\prime}, \phi_{9,5}, \phi_{9,7}\right)$,

$\left(\phi_{1,24}, \phi_{2,12}, \phi_{8,9}^{\prime \prime}, \phi_{8,12}, \phi_{9,8}, \phi_{9,10}\right)$.

6.12. $W=G_{28}$. Le groupe $G_{28}=W\left(F_{4}\right)$ intervient comme groupe relatif dans les groupes réductifs de type $E_{6}$ et $E_{8}$. Les blocs non triviaux sont :

Dans $E_{6}:\left(\phi_{1,12}^{\prime}, \phi_{2,4}^{\prime \prime}, \phi_{9,2}, \phi_{8,3}^{\prime}\right),\left(\phi_{1,12}^{\prime \prime}, \phi_{2,16}^{\prime}, \phi_{9,10}, \phi_{8,9}^{\prime \prime}\right),\left(\phi_{4,8}, \phi_{6,6}^{\prime}, \phi_{6,6}^{\prime \prime}, \phi_{12,4}, \phi_{16,5}\right)$.

Dans $E_{8}:\left(\phi_{4,8}, \phi_{6,6}^{\prime}, \phi_{6,6}^{\prime \prime}, \phi_{12,4}, \phi_{16,5}\right)$.

6.13. $W=G_{31}$. Le groupe $G_{31}$ intervient comme groupe relatif dans les groupes réductifs de type $E_{8}$ (cf. [21, Prop. 7.1]). Les blocs non triviaux sont :

$\left(\phi_{4,1}, \phi_{4,7}\right),\left(\phi_{10,2}, \phi_{10,6}\right),\left(\phi_{20,3}, \phi_{20,5}\right),\left(\phi_{20,7}, \phi_{20,13}^{\prime}\right),\left(\phi_{36,5}, \phi_{36,7}\right),\left(\phi_{40,7}, \phi_{40,9}\right)$,

$\left(\phi_{40,13}, \phi_{40,15}\right),\left(\phi_{36,15}, \phi_{36,17}\right),\left(\phi_{20,13}^{\prime \prime}, \phi_{20,19}\right),\left(\phi_{20,21}, \phi_{20,23}\right),\left(\phi_{10,26}, \phi_{10,30}\right)$,

$\left(\phi_{4,31}, \phi_{4,37}\right),\left(\phi_{10,12}, \phi_{15,8}^{\prime}, \phi_{15,8}^{\prime \prime}, \phi_{30,4}, \phi_{40,6}\right),\left(\phi_{10,24}, \phi_{15,20}^{\prime}, \phi_{15,20}^{\prime \prime}, \phi_{30,16}, \phi_{40,18}\right)$,

$\left(\phi_{6,18}, \phi_{6,14}, \phi_{16,16}, \phi_{24,14}, \phi_{24,6}, \phi_{30,10}^{\prime}, \phi_{30,10}^{\prime \prime}, \phi_{36,10}, \phi_{40,10}, \phi_{40,14}, \phi_{64,9}, \phi_{64,11}\right)$.

6.14. $W=G_{32}$. Le groupe $G_{32}$ intervient comme groupe relatif dans les groupes réductifs de type $E_{8}$ (cf. [21, Prop. 7.1]). Les blocs non triviaux sont :

$\left(\phi_{64,8}, \phi_{64,11}\right),\left(\phi_{64,18}, \phi_{64,21}\right)$,

$\left(\phi_{4,11}, \phi_{6,8}, \phi_{10,2}\right),\left(\phi_{4,51}, \phi_{6,48}, \phi_{10,42}\right),\left(\phi_{20,13}, \phi_{40,10}, \phi_{60,7}\right),\left(\phi_{20,25}, \phi_{40,22}, \phi_{60,19}\right)$,

$\left(\phi_{5,12}, \phi_{15,6}, \phi_{20,3}\right),\left(\phi_{5,44}, \phi_{15,38}, \phi_{20,35}\right),\left(\phi_{10,10}, \phi_{20,7}, \phi_{30,4}\right),\left(\phi_{10,34}, \phi_{20,31}, \phi_{30,28}\right)$,

$\left(\phi_{20,17}, \phi_{40,14}, \phi_{60,11}^{\prime \prime}\right),\left(\phi_{20,21}, \phi_{40,18}, \phi_{60,15}^{\prime \prime}\right),\left(\phi_{20,12}, \phi_{60,12}, \phi_{80,9}\right)$,

$\left(\phi_{20,20}, \phi_{60,20}, \phi_{80,17}\right),\left(\phi_{30,12}^{\prime}, \phi_{30,12}^{\prime \prime}, \phi_{60,9}\right),\left(\phi_{30,20}^{\prime}, \phi_{30,20}^{\prime \prime}, \phi_{60,17}\right)$,

$\left(\phi_{4,21}, \phi_{20,9}^{\prime}, \phi_{20,9}^{\prime \prime}, \phi_{24,6}, \phi_{36,7}, \phi_{36,5}\right),\left(\phi_{4,41}, \phi_{20,29}^{\prime}, \phi_{20,29}^{\prime \prime}, \phi_{24,26}, \phi_{36,27}, \phi_{36,25}\right)$,

$\left(\phi_{5,20}, \phi_{10,14}, \phi_{15,8}, \phi_{40,8}, \phi_{45,6}, \phi_{45,10}\right),\left(\phi_{5,36}, \phi_{10,30}, \phi_{15,24}, \phi_{40,24}, \phi_{45,22}, \phi_{45,26}\right)$,

$\left(\phi_{1,40}, \phi_{6,28}, \phi_{15,16}, \phi_{15,22}, \phi_{20,19}, \phi_{20,16}, \phi_{24,16}, \phi_{36,17}, \phi_{36,15}, \phi_{45,14}, \phi_{45,18}, \phi_{60,13}\right.$,

$\left.\phi_{60,16}, \phi_{64,16}, \phi_{64,13}, \phi_{80,13}, \phi_{81,12}, \phi_{81,14}, \phi_{81,10}\right)$.

\section{RÉFÉRENCES}

[1] D. Alvis Et G. Lusztig, The representations and generic degrees of the Hecke algebra of type $H_{4}$, J. reine angew. Math. 336 (1982), 201-212.

[2] D. Bessis, Sur le corps de définition d'un groupe de réflexions complexe, Comm. in Algebra 25 (1997), 2703-2716. 
[3] M. Broué ET S. Kim, Familles de caractères des algèbres de Hecke cyclotomiques, Advances in Math. 172 (2002), 53-136.

[4] M. Broué et G. Malle, Zyklotomische Heckealgebren, Astérisque 212 (1993), 119-189.

[5] M. Broué et G. Malle, Generalized Harish-Chandra theory, in : Representations of reductive groups, Cambridge University Press, Cambridge, 1998, pp. 85-103.

[6] M. Broué, G. Malle et J. Michel, Generic blocks of finite reductive groups, Astérisque 212 (1993), 7-92

[7] M. Broué, G. Malle et J. Michel, Towards spetses I, Transform. Groups 4 (1999), 157218.

[8] M. Broué, G. Malle et R. Rouquier, Complex reflection groups, braid groups, Hecke algebras, J. reine angew. Math. 500 (1998), 127-190

[9] M. Broué et J. Michel, Sur certains éléments réguliers des groupes de Weyl et les variétés de Deligne-Lusztig associées, in : M. Cabanes (ed.), Finite reductive groups, related structures and representations, Progress in Math. 141, Birkhäuser, 1997, pp. 73-139.

[10] M. Geck, G. Hiss, F. Lübeck, G. Malle and G. Pfeiffer, CheVIE - A system for computing and processing generic character tables. Appl. Algebra Engrg. Comm. Comput. 7 (1996), 175-210.

[11] M. Geck et G. Pfeiffer, Characters of finite Coxeter groups and Iwahori-Hecke algebras, Oxford University Press, Oxford, 2000.

[12] M. Geck et R. Rouquier, Centers and simple modules for Iwahori-Hecke algebras, in : M. Cabanes (ed.), Finite reductive groups, related structures and representations, Progress in Math. 141, Birkhäuser, 1997, pp. 251-272.

[13] A. Gyoja, Cells and modular representations of Hecke algebras, Osaka J. Math. 33 (1996), 307-341.

[14] G. Lusztig, A class of irreducible representations of a Weyl group. II, Indag. Math. 44 (1982), 219-226.

[15] G. LuszTig, Characters of reductive groups over a finite field, Annals Math. Studies 107, Princeton University Press, Princeton, 1984.

[16] G. LuszTig, Hecke algebras with unequal parameters, CRM Monographs, Amer. Math. Soc., Providence, 2003.

[17] G. Malle, Unipotente Grade imprimitiver komplexer Spiegelungsgruppen, J. Algebra 177 (1995), 768-826.

[18] G. Malle, Degrés relatifs des algèbres cyclotomiques associées aux groupes de réflexions complexes de dimension deux, in : M. Cabanes (ed.), Finite reductive groups, related structures and representations, Progress in Math. 141, Birkhäuser, 1997, pp. 311-332.

[19] G. Malle, Spetses, Doc. Math. J. DMV Extra Volume ICM II (1998), 87-96.

[20] G. Malle, On the rationality and fake degrees of characters of cyclotomic algebras, J. Fac. Sci. Univ. Tokyo 6 (1999), 647-677.

[21] G. Malle, On the generic degrees of cyclotomic algebras, Represent. Theory 4 (2000), 342369

[22] R. Rouquier, Familles et blocs d'algèbres de Hecke, C. R. Acad. Sci. Paris 329 (1999), $1037-1042$

G.M. : Fachbereich Mathematik/Informatik, Universität Kassel, Heinrich-PlettStr. 40, D-34132 Kassel, Germany.

E-mail address: malle@mathematik.uni-kassel.de

R.R. : UMR 7586 du CNRS et UFR de Mathématiques, Université Denis Diderot, Case 7012, 2 Place Jussieu, F-75251 Paris Cedex 05, France.

E-mail address: rouquier@math.jussieu.fr 